\title{
Modified Rayleigh conjecture method and its applications
}

\author{
Alexander G. Ramm ${ }^{\mathrm{a}, *}$, Semion Gutman ${ }^{\mathrm{b}}$ \\ ${ }^{a}$ Department of Mathematics, Kansas State University, Manhattan, KS 66506-2602, USA \\ ${ }^{\mathrm{b}}$ Department of Mathematics, University of Oklahoma, Norman, OK 73019, USA
}

Received 28 March 2007; accepted 5 April 2007

\begin{abstract}
The Rayleigh conjecture about convergence up to the boundary of the series representing the scattered field in the exterior of an obstacle $D$ is widely used by engineers in applications. However this conjecture is false for some obstacles. AGR introduced the Modified Rayleigh Conjecture (MRC), which is an exact mathematical result. In this paper we present the theoretical basis for the MRC method for 2D and 3D obstacle scattering problems, for static problems, and for scattering by periodic structures. We also present successful numerical algorithms based on the MRC for various scattering problems. The MRC method is easy to implement for both simple and complex geometries. It is shown to be a viable alternative for other obstacle scattering methods. Various direct and inverse scattering problems require finding global minima of functions of several variables. The Stability Index Method (SIM) combines stochastic and deterministic method to accomplish such a minimization.
\end{abstract}

(C) 2007 Elsevier Ltd. All rights reserved.

MSC: 35J05; 65M99; 78A40

Keywords: Obstacle scattering; Modified Rayleigh conjecture; Stability index method

\section{Introduction}

In this paper we review our recent results on the Modified Rayleigh Conjecture (MRC) method. The method is applied to multidimensional obstacle scattering problems, as well as to scattering by periodic structures. Also we discuss an application of the MRC to static problems, and preliminary results on inverse obstacle scattering by MRC. Numerical results illustrate the performance of various MRC algorithms. The paper concludes with a presentation of the Stability Index Method (SIM) for global minimization.

The basic theoretical foundation of the method was developed in [27]. The MRC has the appeal of an easy implementation for obstacles of complicated geometry, e.g. having edges and corners. In our numerical experiments the method has shown itself to be a competitive alternative to the BIEM (boundary integral equations method), see [13]. Also, unlike the BIEM, one can apply the algorithm to different obstacles with very little additional effort. A similar method is discussed in [10]

We formulate the obstacle scattering problem in a 3D setting with the Dirichlet boundary condition, but the method can also be used for the Neumann boundary condition, corresponding to acoustically hard obstacles, and the Robin boundary condition.

\footnotetext{
* Corresponding author. Tel.: +1 785532 0580; fax: +1 7855320546.

E-mail addresses: ramm@math.ksu.edu (A.G. Ramm), sgutman@ou.edu (S. Gutman).
} 
Consider a bounded domain $D \subset \mathbb{R}^{3}$, with a Lipschitz boundary $S$. Denote the exterior domain by $D^{\prime}=\mathbb{R}^{3} \backslash D$. Let $\alpha, \alpha^{\prime} \in S^{2}$ be unit vectors, where $S^{2}$ is the unit sphere in $\mathbb{R}^{3}$.

The acoustic wave scattering problem by an acoustically soft obstacle $D$ consists in finding the (unique) solution to the problem (1.1) and (1.2):

$$
\begin{aligned}
& \left(\nabla^{2}+k^{2}\right) u=0 \quad \text { in } D^{\prime}, u=0 \text { on } S, \\
& u=u_{0}+A\left(\alpha^{\prime}, \alpha\right) \frac{\mathrm{e}^{\mathrm{i} k r}}{r}+o\left(\frac{1}{r}\right), \quad r:=|x| \rightarrow \infty, \alpha^{\prime}:=\frac{x}{r} .
\end{aligned}
$$

Here $u_{0}:=\mathrm{e}^{\mathrm{i} k \alpha \cdot x}$ is the incident field, $v:=u-u_{0}$ is the scattered field, $A\left(\alpha^{\prime}, \alpha\right)$ is called the scattering amplitude, its $k$-dependence is not shown, $k>0$ is the wavenumber. The scattered field $v$ is an outgoing solution of the Helmholtz differential equation (1.1), that is, a solution which satisfies the radiation condition

$$
\lim _{r \rightarrow \infty} \int_{|x|=r}\left|\frac{\partial v}{\partial|x|}-\mathrm{i} k v\right|^{2} \mathrm{~d} s=0 .
$$

Denote

$$
A_{\ell}(\alpha):=\int_{S^{2}} A\left(\alpha^{\prime}, \alpha\right) \overline{Y_{\ell}\left(\alpha^{\prime}\right)} \mathrm{d} \alpha^{\prime},
$$

where $Y_{\ell}(\alpha)$ are the orthonormal spherical harmonics, $Y_{\ell}=Y_{\ell m},-\ell \leq m \leq \ell$.

Let a ball $B_{R}:=\{x:|x| \leq R\}$ contain the obstacle $D$. Let $h_{\ell}(r)$ be the spherical Hankel functions, normalized so that $h_{\ell}(r) \sim \frac{\mathrm{e} \text { ikr }}{r}$ as $r \rightarrow+\infty$. In the region $r>R$ the solution to (1.1) and (1.2) is:

$$
u(x, \alpha)=\mathrm{e}^{\mathrm{i} k \alpha \cdot x}+\sum_{\ell=0}^{\infty} A_{\ell}(\alpha) \Psi_{\ell}(x), \quad \Psi_{\ell}(x):=Y_{\ell}\left(\alpha^{\prime}\right) h_{\ell}(k r), r>R, \alpha^{\prime}=\frac{x}{r},
$$

where $r=|x|$, the sum includes the summation with respect to $m,-\ell \leq m \leq \ell$, and $A_{\ell}(\alpha)$ are defined in (1.4), see $[26,25]$.

The Rayleigh conjecture $(R C)$ is: the series (1.5) converges up to the boundary $S$ (originally RC dealt with periodic structures, gratings). This conjecture is false for many obstacles, but is true for some $[3,22,28]$. For example, if $n=2$ and $D$ is an ellipse, then the series analogous to (1.5) converges in the region $|x|>a$, where $2 a$ is the distance between the foci of the ellipse [3]. In the engineering literature there are numerical algorithms based on the Rayleigh conjecture. These algorithms use projection methods and are reported to be unstable. Moreover, no error estimate has been obtained for such algorithms. These algorithms cannot converge for arbitrary obstacles, because the Rayleigh conjecture is false for some obstacles.

Our aim is to give a formulation of a Modified Rayleigh Conjecture (MRC) which holds for any Lipschitz obstacle and can be used in numerical solution of direct and inverse scattering problems. In other words, while the MRC still has the word "conjecture" in its name, it is a proven mathematical result for the scattered field in the exterior domain $D^{\prime}$. In contrast to algorithms based on the invalid Rayleigh conjecture, the MRC-based algorithms, like the ones described here, converge and an error estimate for the approximate solution they yield has been obtained in [27] (see also [34], Chapter 12). This error estimate is sharp in the order $\epsilon$.

\section{Modified Rayleigh conjecture}

What we call the Modified Rayleigh Conjecture (MRC) is actually the following Theorems 2.1, see [27], and 2.3, see [16]. We denote by $H_{\mathrm{loc}}^{m}\left(D^{\prime}\right)$ the set of functions from the Sobolev space $H^{m}(\tilde{D})$ for any compact strictly inner subdomain $\tilde{D}$ of $D^{\prime}$, so that the distance from $\tilde{D}$ to $S$ is positive, $\operatorname{dist}(\tilde{D}, S)>0$.

Theorem 2.1. Let $v=u-u_{0}$ be the scattered field, where $u$ is the solution to (1.1) and (1.2). Then there exists a positive integer $L=L(\epsilon)$ and the coefficients $c_{\ell}=c_{\ell}(\epsilon), 0 \leq \ell \leq L(\epsilon)$ such that 
(i)

$$
\left\|u_{0}+v_{\epsilon}\right\|_{L^{2}(S)} \leq \epsilon
$$

where

$$
v_{\epsilon}(x)=\sum_{\ell=0}^{L(\epsilon)} c_{\ell}(\epsilon) \Psi_{\ell}(x) .
$$

(ii)

$$
\left\|v_{\epsilon}-v\right\|_{L^{2}(S)} \leq \epsilon
$$

and

$$
\left\|v_{\epsilon}-v\right\|=O(\epsilon), \quad \epsilon \rightarrow 0
$$

where

(iii)

$$
\|\cdot\|=\|\cdot\|_{H_{\mathrm{loc}}^{m}\left(D^{\prime}\right)}+\|\cdot\|_{L^{2}\left(D^{\prime} ;(1+|x|)^{-\gamma}\right)},
$$

$\gamma>1, m>0$ is an arbitrary integer.

$$
c_{\ell}(\epsilon) \rightarrow A_{\ell}, \quad \text { as } \epsilon \rightarrow 0, \forall \ell,
$$

where $A_{\ell}:=A_{\ell}(\alpha)$ is defined in (1.4).

Proof. First, we prove item (i). Then we establish Lemma 2.2, and continue with the proof of (ii) and (iii).

(i) Without loss of generality we can assume that the origin is an interior point of the domain $D$. To establish (2.1) it is sufficient to show that

$$
H:=\overline{\operatorname{span}}\left\{\Psi_{\ell}(s): 0 \leq \ell<\infty, s \in S\right\}=L^{2}(S) .
$$

Suppose that there exists $p \in L^{2}(S), p \neq 0$, such that $p \perp H$ in $L^{2}(S)$. Define the single-layer potential by

$$
W(y)=\int_{S} \frac{\mathrm{e}^{\mathrm{i} k|s-y|}}{|s-y|} p(s) \mathrm{d} s, \quad y \in \mathbb{R}^{3},
$$

where $\mathrm{d} s$ is the surface area element. Let $U \subset D$ be a ball centered at the origin. Then the addition theorem for the fundamental solution implies that $W(y)=0$ for any $y \in U$.

By the unique continuation principle $W \equiv 0$ in $D$. In particular $W=0$ on the boundary $S$. Since $W$ is an outgoing solution of $\left(\nabla^{2}+k^{2}\right) W=0$ in $D^{\prime}$ with $W=0$ on $S$, one concludes from the uniqueness of solutions to the Dirichlet problem in $D^{\prime}$ that $W \equiv 0$ in $\mathbb{R}^{3}$. Finally, the jump properties of the normal derivative of the single-layer potential imply that $p=0$ in $L^{2}(S)$. We have followed the argument from [33], p. 160.

Lemma 2.2. Given $g \in L_{2}(S)$, let $w$ be the outgoing solution of the exterior Dirichlet problem $\left(\nabla^{2}+k^{2}\right) w=0$, in $D^{\prime}$ with $w=g$ on $S$. Then there exists a constant $C>0$, independent of $w$, such that

$$
\|w\| \leq C\|g\|_{L^{2}(S)},
$$

where $\|\cdot\|:=\|\cdot\|_{H_{\mathrm{loc}}^{m}\left(D^{\prime}\right)}+\|\cdot\|_{L^{2}\left(D^{\prime} ;(1+|x|)^{-\gamma}\right)}, \gamma>1, m>0$ is an arbitrary integer, and $H^{m}$ is the Sobolev space.

Proof. Let $G$ be the Dirichlet Green's function of the Laplacian in $D^{\prime}$ :

$$
\begin{aligned}
& \left(\nabla^{2}+k^{2}\right) G=-\delta(x-y) \quad \text { in } D^{\prime}, G=0 \text { on } S, \\
& \lim _{r \rightarrow \infty} \int_{|x|=r}\left|\frac{\partial G}{\partial|x|}-\mathrm{i} k G\right|^{2} \mathrm{~d} s=0 .
\end{aligned}
$$

Let $N$ be the unit normal to $S$ pointing into $D^{\prime}$. By Green's formula one has

$$
w(x)=\int_{S} g(s) \frac{\partial G}{\partial N}(x, s) \mathrm{d} s, \quad x \in D^{\prime} .
$$


The estimate for the $H_{\mathrm{loc}}^{m}\left(D^{\prime}\right)$-norm part of (2.7) follows from this representation and from the Cauchy inequality:

$$
\left|D^{(j)} w(x)\right| \leq\|g\|_{L^{2}(S)}\left\|\frac{\partial D_{x}^{(j)} G}{\partial N}(x, s)\right\| \leq c(x)\|g\|_{L^{2}(S)},
$$

where $c(x) \leq c(d)$ for all $x \in D^{\prime}$ such that the distance $\operatorname{dist}(x, S) \geq d>0$.

For the $L^{2}$-weighted norm part of (2.7) let $R>0$ be such that $D \subset B_{R}=\left\{x \in \mathbb{R}^{3}:|x|<R\right\}$. Let $D_{R}^{\prime}=B_{R} \backslash D$, and $S_{R}$ be the boundary of $B_{R}$. The estimate

$$
\left|\frac{\partial G}{\partial N}(x, s)\right| \leq \frac{c}{1+|x|}, \quad|x| \geq R,
$$

and formula (2.10) imply

$$
\|w\|_{L^{2}\left(S_{R}\right)} \leq c\|g\|_{L^{2}(S)},
$$

where here and in the sequel $c$ and $C$ denote various constants. Also, using the Cauchy inequality, formula (2.10), inequality (2.11) and the assumption $\gamma>1$, one gets

$$
\|w\|_{L^{2}\left(|x|>R ;(1+|x|)^{-\gamma}\right)} \leq c\|g\|_{L^{2}(S)}\left\|\frac{1}{(1+|x|)^{\gamma+1}}\right\|_{L^{2}(|x|>R)} \leq c\|g\|_{L^{2}(S)} .
$$

To get the estimate for $\|w\|_{L^{2}\left(D_{R}^{\prime}\right)}$ choose $R$ such that $k^{2}$ is not a Dirichlet eigenvalue of $-\Delta$ in $D_{R}^{\prime}$. Then ([20], p. 189):

$$
\|w\|_{H^{m}\left(D_{R}^{\prime}\right)} \leq c\left[\left\|\left(\Delta+k^{2}\right) w\right\|_{\mathcal{H}^{m-2}\left(D_{R}^{\prime}\right)}+\|w\|_{H^{m-0.5}\left(S_{R}\right)}+\|w\|_{H^{m-0.5}(S)}\right] .
$$

The space $\mathcal{H}$ in the first term of the right-hand side in (2.14) is different from the usual Sobolev space, but this term is equal to zero anyway because $\left(\Delta+k^{2}\right) w=0$.

Let $m=0.5$ in (2.14). Then

$$
\|w\|_{H^{0.5}\left(D_{R}^{\prime}\right)} \leq c\left[\|w\|_{L^{2}\left(S_{R}\right)}+\|w\|_{L^{2}(S)}\right] .
$$

Since $w=g$ on $S$, then (2.12) and (2.15) imply

$$
\|w\|_{L^{2}\left(D_{R}^{\prime}\right)} \leq c\|g\|_{L^{2}(S)} \text {. }
$$

Proof of Theorem 2.1, continued.

(ii) Inequality (2.3) is the same as (2.1), since $v=-u_{0}$ on $S$. Estimate (2.4) follows from (2.3) and Lemma 2.2.

(iii) Inequality (2.3) yields the convergence of $v_{\epsilon}$ to $v$ in the norm $\|\cdot\|_{L^{2}(S)}$. By (2.12) $\left\|v_{\epsilon}-v\right\|_{L^{2}\left(S_{R}\right)} \rightarrow 0$, as $\epsilon \rightarrow 0$. On $S_{R}$ one has $v=\sum_{\ell=0}^{\infty} A_{\ell}(\alpha) \Psi_{\ell}$ and $v_{\epsilon}=\sum_{\ell=0}^{L(\epsilon)} c_{\ell} \Psi_{\ell}$. Multiply $v_{\epsilon}\left(R, \alpha^{\prime}\right)-v\left(R, \alpha^{\prime}\right)$ by $\overline{Y_{\ell}\left(\alpha^{\prime}\right)}$, integrate over $S^{2}$ and then let $\epsilon \rightarrow 0$. The result is (iii), and the proof of Theorem 2.1 is completed.

The difference between RC and MRC is: (2.1) does not hold if one replaces $v_{\epsilon}$ by $\sum_{\ell=0}^{L} A_{\ell}(\alpha) \Psi_{\ell}$, and lets $L \rightarrow \infty$ (instead of letting $\epsilon \rightarrow 0$ ). Indeed, the series $\sum_{\ell=0}^{\infty} A_{\ell}(\alpha) \Psi_{\ell}$ diverges at some points of the boundary for many obstacles. Note also that the coefficients in (2.2) depend on $\epsilon$, so (2.2) is not a partial sum of a series.

For the Neumann boundary condition one minimizes

$$
\left\|\frac{\partial\left[u_{0}+\sum_{\ell=0}^{L} c_{\ell} \psi_{\ell}\right]}{\partial N}\right\|_{L^{2}(S)}
$$

with respect to $c_{\ell}$, and obtains essentially the same results.

According to Theorem 2.1 the computation of the outgoing solution to (1.1) and (1.2) is reduced to the approximation of the boundary values in (1.1) by the linear combinations of the functions $\Psi_{\ell}$ restricted to the boundary $S$. A direct implementation of the above algorithm is efficient for domains $D$ not very different from a circle, e.g. for an 
ellipse with a small eccentricity, but it fails for more complicated regions. The numerical difficulties happen because the spherical Hankel functions $h_{l}$ with large values of $l$ are bigger than $h_{l}$ with small values of $l$ by many orders of magnitude. A finite precision of numerical computations makes it necessary to keep the values of $L$ not too high, e.g. $L \leq 20$. This restriction can be remedied by the following modification of the above algorithm, see $[13,16]$ :

Theorem 2.3. Let $v:=u-u_{0}$, where $u$ is the solution to (1.1) and (1.2). Let $\epsilon>0$, and $L$ be a nonnegative integer. Suppose $U$ is an open subset of $D$.

Then there exist a finite subset $\left\{z_{1}, z_{2}, \ldots, z_{J}\right\} \subset U$, and the coefficients $c_{\ell}\left(\epsilon, z_{j}\right), 0 \leq \ell \leq L, 1 \leq j \leq J=$ $J(\epsilon)$, such that the following inequalities (2.17) and (2.20) hold:

(i)

$$
\left\|u_{0}+v_{\epsilon}\right\|_{L^{2}(S)} \leq \epsilon
$$

where

$$
v_{\epsilon}(x):=\sum_{j=1}^{J} \sum_{\ell=0}^{L} c_{\ell}\left(\epsilon, z_{j}\right) \psi_{\ell}\left(x, z_{j}\right),
$$

and

$$
\psi_{\ell}(x, z)=Y_{\ell}\left(\alpha^{\prime}\right) h_{\ell}(k|x-z|), \quad \alpha^{\prime}=\frac{x-z}{|x-z|}, z \in D, x \in \mathbb{R}^{3} \backslash D .
$$

(ii)

$$
\left\|v_{\epsilon}-v\right\|_{L^{2}(S)} \leq \epsilon
$$

and

$$
\left\|v_{\epsilon}-v\right\|=O(\epsilon), \quad \epsilon \rightarrow 0,
$$

where

$$
\|\cdot\|=\|\cdot\|_{H_{\mathrm{loc}}^{m}\left(D^{\prime}\right)}+\|\cdot\|_{L^{2}\left(D^{\prime} ;(1+|x|)^{-\gamma}\right)},
$$

$\gamma>1, m>0$ is an arbitrary integer, and $H^{m}$ is the Sobolev space.

Proof. (i) Note that in Theorem 2.1 we had $L=L(\epsilon)$, while now we have $L$ fixed and $J=J(\epsilon)$. But the proof of Theorem 2.3 is similar to that of Theorem 2.1. Let $\left\{z_{j}\right\}_{j=1}^{\infty}$ be a countable dense subset of $U$. To establish (2.17) it is sufficient to show that

$$
H:=\overline{\operatorname{span}}\left\{\psi_{\ell}\left(s, z_{j}\right): 0 \leq \ell \leq L, j=1,2, \ldots\right\}=L^{2}(S) .
$$

Suppose that there exists $p \in L^{2}(S), p \neq 0$ such that $p \perp H$ in $L^{2}(S)$. Define the single-layer potential by

$$
W(y)=\int_{S} \frac{\mathrm{e}^{\mathrm{i} k|s-y|}}{|s-y|} p(s) \mathrm{d} s, \quad y \in \mathbb{R}^{3} .
$$

Then

$$
W\left(z_{j}\right)=\int_{S} \psi_{0}\left(s, z_{j}\right) p(s) \mathrm{d} s=0
$$

for $j=1,2, \ldots$.

The continuity of the single-layer potential in $\mathbb{R}^{3}$ implies that $W(y)=0$ for all $y \in U$. The rest of the proof is the same as in Theorem 2.1.

Remark. Functions $\left\{\Psi_{l}\right\}_{\ell=0}^{\infty}$ are linearly independent on $S$. Indeed, if some finite combination of these functions vanishes on $S$, then it also vanishes in the exterior domain $D^{\prime}$, since such a combination is an outgoing solution of the exterior Dirichlet problem with zero boundary conditions on $S$. In particular, such a combination also vanishes on $S_{R}$. Since the spherical functions are orthogonal on $S_{R}$, it implies that such a combination must be trivial.

See Section 6 and Section 7 for an extension of the MRC method to static problems, and to scattering by periodic structures, respectively. 


\section{Iterative MRC algorithms}

Let $z$ be a point in the interior of the obstacle $D$, and $x \in \mathbb{R}^{3} \backslash D$. Recall that

$$
\psi_{\ell}(x, z)=Y_{\ell}\left(\alpha^{\prime}\right) h_{\ell}(k|x-z|),
$$

where $h_{\ell}(r)$ are the spherical Hankel functions, normalized so that $h_{\ell}(r) \sim \frac{\mathrm{e}^{\mathrm{i} k r}}{r}$ as $r \rightarrow+\infty$.

Noniterative MRC.

In this MRC implementation one chooses a set of interior points $H=\left\{x_{j} \in D, j=1,2, \ldots, J, J>0\right\}$ and minimizes

$$
\Phi(\mathbf{c})=\left\|u_{0}(s)+\sum_{j=1}^{J} \sum_{\ell=0}^{L} c_{\ell, j} \psi_{\ell}\left(s, x_{j}\right)\right\|_{L^{2}(S)},
$$

over $\mathbf{c} \in \mathbb{C}^{N}$, where $\mathbf{c}=\left\{c_{\ell, j}\right\}$. That is, the total field $u(s)=u_{0}(s)+v(s)$ is desired to be as close to zero as possible at the boundary $S$, to satisfy the required condition for soft scattering. If the resulting residual $r^{\min }=\min \Phi$ is smaller than the prescribed tolerance $\epsilon$, then the procedure is finished, and the sought scattered field is

$$
v_{\epsilon}(x)=\sum_{j=1}^{J} \sum_{\ell=0}^{L} c_{\ell, j} \psi_{\ell}\left(x, x_{j}\right), \quad x \in D^{\prime} .
$$

If the residual $r^{\mathrm{min}}>\epsilon$ then the method fails. This approach, which can be called a Multi-point MRC, is justified by Theorem 2.3. See $[13,32,10]$ for details and results of numerical experiments. The results show that the method is very efficient for domains $D$ of a nearly spherical shape, i.e. without elongated parts. Clearly, the only limitation in this method is the computer resources. The method becomes impractical for large sets of interior points $H$.

To remedy this situation one can use iterative MRC implementations, of which we describe the one based on a random choice of interior points, and another one based on an optimal choice of such points.

Iterative MRC with a random choice of points.

Informally, the Random Multi-point MRC algorithm can be described as follows.

Fix a $J>0$. Let $x_{j}, j=1,2, \ldots, J$ be a batch of points randomly chosen inside the obstacle $D$.

Let $g(s)=u_{0}(s), s \in S$, and minimize the discrepancy

$$
\Phi(\mathbf{c})=\left\|g(s)+\sum_{j=1}^{J} \sum_{\ell=0}^{L} c_{\ell, j} \psi_{\ell}\left(s, x_{j}\right)\right\|_{L^{2}(S)}
$$

over $\mathbf{c} \in \mathbb{C}^{N}$, where $\mathbf{c}=\left\{c_{\ell, j}\right\}$. That is, the total field $u(s)=g(s)+v(s)$ is desired to be as close to zero as possible at the boundary $S$, to satisfy the required condition for soft scattering. If the resulting residual $r^{\min }=\min \Phi$ is smaller than the prescribed tolerance $\epsilon$, then the procedure is finished, and the sought scattered field is

$$
v_{\epsilon}(x)=\sum_{j=1}^{J} \sum_{\ell=0}^{L} c_{\ell, j} \psi_{\ell}\left(x, x_{j}\right), \quad x \in D^{\prime} .
$$

If, on the other hand, the residual $r^{\min }>\epsilon$, then we continue by trying to improve on the already obtained fit in (3.3). Adjust the field on the boundary by letting $g(s):=g(s)+v_{\epsilon}(s), s \in S$. Create another batch of $J$ points randomly chosen in the interior of $D$, and minimize (3.3) with this new $g(s)$. Continue with the iterations until the required tolerance $\epsilon$ on the boundary $S$ is attained. In each iteration accumulate new interior points $x_{j}$ and the corresponding best fit coefficients $c_{\ell, j}$. After the desired tolerance is reached, the sought scattered field $v_{\epsilon}$ is computed anywhere in $D^{\prime}$.

Here is a precise description of the algorithm.

Random Multi-point MRC.

For $x_{j} \in D$, and $\ell \geq 0$ functions $\psi_{\ell}\left(x, x_{j}\right)$ are defined as in (3.1).

1. Initialization. Fix $\epsilon>0, L \geq 0, J>0, N_{\max }>0$. Let $n=0$, and $g(s)=u_{0}(s), s \in S$. 
2. Iteration.

(a) Let $n:=n+1$. Randomly choose $J$ points $x_{j}^{(n)} \in D, j=1,2, \ldots, J$.

(b) Minimize

$$
\Phi(\mathbf{c})=\left\|g(s)+\sum_{j=1}^{J} \sum_{\ell=0}^{L} c_{\ell, j} \psi_{\ell}\left(s, x_{j}^{(n)}\right)\right\|_{L^{2}(S)}
$$

over $\mathbf{c} \in \mathbb{C}^{N}$, where $\mathbf{c}=\left\{c_{\ell, j}\right\}$.

Let the minimum of $\Phi$ be attained at $\mathbf{c}^{(n)}=\left\{c_{\ell, j}^{(n)}\right\}, j=1,2, \ldots, J$, and the minimal value of $\Phi$ be $r^{\min }$.

3. Stopping criterion.

(a) If $r^{\mathrm{min}} \leq \epsilon$, then stop. Compute the approximate scattered field anywhere in $D^{\prime}$ by

$$
v_{\epsilon}(x):=\sum_{k=1}^{n} \sum_{j=1}^{J} \sum_{\ell=0}^{L} c_{\ell, j}^{(k)} \psi_{\ell}\left(x, x_{j}^{(k)}\right), \quad x \in D^{\prime} .
$$

(b) If $r^{\min }>\epsilon$, and $n \neq N_{\max }$, let

$$
g(s):=g(s)+\sum_{j=1}^{J} \sum_{\ell=0}^{L} c_{\ell, j}^{(n)} \psi_{\ell}\left(s, x_{j}^{(n)}\right), \quad x \in S
$$

and repeat the iterative step (2).

(c) If $r^{\min }>\epsilon$, and $n=N_{\max }$, then the procedure failed.

Numerical experiments based on this method are presented in the next section. The method is relatively slow, and it can be improved by choosing the interior points in some optimal way.

Iterative MRC with an optimal choice of points.

In this case the interior points $z_{1}, z_{2}, \ldots$ in $D$ are chosen one at a time, and their placement is not random. Rather, the discrepancy $\Psi$ is minimized not only with respect to the coefficients $\mathbf{c}$, but also with respect to the position of these points $z_{j}$.

Let $g_{1}(s)=u_{0}(s)=u_{0}(s, \alpha), s \in S$.

Minimize

$$
\Phi\left(z_{1}, \mathbf{c}\left(z_{1}\right)\right):=\min _{z \in D} \min _{\mathbf{c} \in \mathbb{C}^{N}}\left\|g_{1}(s)+\sum_{\ell=0}^{L} c_{\ell} \psi_{\ell}(s, z)\right\|_{L^{2}(S)},
$$

where $\mathbf{c}=\left\{c_{\ell}\right\}=\left\{c_{\ell m}\right\}_{0 \leq \ell \leq L,-\ell \leq m \leq \ell}, L \geq 0$ is a fixed integer, and $\sum_{\ell=0}^{L}:=\sum_{\ell=0}^{L} \sum_{m=-\ell}^{\ell}$. Let

$$
v_{1}(x)=\sum_{\ell=0}^{L} c_{\ell}\left(z_{1}\right) \psi_{\ell}\left(x, z_{1}\right), \quad c_{\ell}\left(z_{1}\right)=c_{\ell}\left(z_{1}, \alpha\right) .
$$

The requirement (3.5) means that the total field $u(s)=g_{1}(s)+v_{1}(s)$ has to be as close to zero as possible on the boundary $S$, so that it approximates best the Dirichlet boundary condition in (1.1). This is achieved by varying the interior point $z \in D$ and choosing the coefficients $\mathbf{c}(z) \in \mathbb{C}^{N}$ giving $g_{1}+v_{1}$ the best fit to zero on the boundary $S$. Let the minimum in (3.5) be attained at $z_{1} \in D$. If the resulting value of the residual $r^{\text {min }}=\Phi\left(z_{1}, \mathbf{c}\left(z_{1}\right)\right)$ is smaller than the prescribed tolerance $\epsilon$, than the procedure is finished. The sought approximate scattered field is $v_{1}(x), x \in D^{\prime}$ (see Theorem 2.3), and the approximate scattering amplitude is

$$
A_{1}\left(\alpha^{\prime}, \alpha\right)=\mathrm{e}^{-\mathrm{i} k \alpha^{\prime} \cdot z_{1}} \sum_{\ell=0}^{L} c_{\ell}\left(z_{1}\right) Y_{\ell}\left(\alpha^{\prime}\right) .
$$

Note that $c_{\ell}\left(z_{1}\right)=c_{\ell}\left(z_{1}, \alpha\right)$.

The expression for $A_{1}\left(\alpha^{\prime}, \alpha\right)$ in (3.7) is obtained from (3.6) by letting $|x| \rightarrow \infty$ in $x=\alpha^{\prime}|x|$, because of our normalization

$$
h_{\ell}(k|x|)=\frac{\mathrm{e}^{\mathrm{i} k|x|}}{|x|}\left\{1+O\left(\frac{1}{|x|}\right)\right\}, \quad|x| \rightarrow \infty,
$$

and $|x-z|=|x|-\alpha^{\prime} \cdot z+O(1 /|x|)$ as $|x| \rightarrow \infty$. 
If, on the other hand, the residual $r^{\mathrm{min}}>\epsilon$, then we continue by trying to improve on the already obtained fit in (3.5) as follows. Adjust the field on the boundary by letting $g_{2}(s)=g_{1}(s)+v_{1}(s), s \in S$, and do the minimization (3.3) with $g_{2}(s)$ instead of $g_{1}(s)$, etc. Continue with the iterations until the required tolerance $\epsilon$ on the boundary $S$ is attained. At the same time keep track of the changing approximate scattered field $v_{n}(x)$, and the scattering amplitude $A_{n}\left(\alpha^{\prime}, \alpha\right)$. In this construction $g_{n+1}=u_{0}+v_{n}$ on $S$. The goal of (3.3) is to obtain $g_{n} \rightarrow 0$ in $L^{2}(S)$ as $n \rightarrow \infty$, yielding $u_{0}+v_{n} \rightarrow 0$ in $L^{2}(S)$. According to Theorem 2.3, this gives an approximate scattered solution $v_{n}$ on $D^{\prime}$ to (1.1) and (1.2).

Here is a precise description of the algorithm.

MRC method with optimal choice of sources.

1. Initialization. Fix $\epsilon>0, L \geq 0, N_{\max }>0$. Let $n=0, v_{0}(x)=0, A_{0}\left(\alpha^{\prime}, \alpha\right)=0$, and $g_{1}(s)=u_{0}(s), s \in S$.

2. Iteration.

(a) Increase the value of $n$ by 1 .

(b) Minimize

$$
\Phi\left(z_{n}, \mathbf{c}\left(z_{n}\right)\right):=\min _{z \in D} \min _{\mathbf{c} \in \mathbb{C}^{N}}\left\|g_{n}(s)+\sum_{\ell=0}^{L} c_{\ell} \psi_{\ell}(s, z)\right\|_{L^{2}(S)},
$$

with the minimal value attained at $z_{n} \in D, \mathbf{c}\left(z_{n}\right) \in \mathbb{C}^{N}$.

(c) Let

$$
\begin{aligned}
& v_{n}(x)=v_{n-1}(x)+\sum_{\ell=0}^{L} c_{\ell}\left(z_{n}\right) \psi_{\ell}\left(x, z_{n}\right), \quad x \in D^{\prime}, \\
& A_{n}\left(\alpha^{\prime}, \alpha\right)=A_{n-1}\left(\alpha^{\prime}, \alpha\right)+\mathrm{e}^{-\mathrm{i} k \alpha^{\prime} \cdot z_{n}} \sum_{\ell=0}^{L} c_{\ell}\left(z_{n}\right) Y_{\ell}\left(\alpha^{\prime}\right),
\end{aligned}
$$

and

$$
g_{n+1}(s)=g_{n}(s)+\sum_{\ell=0}^{L} c_{\ell}\left(z_{n}\right) \psi_{\ell}\left(s, z_{n}\right), \quad s \in S,
$$

that is $g_{n+1}(s)=u_{0}(s)+v_{n}(s), s \in S$.

(d) Let

$$
r^{\min }:=\Phi\left(z_{n}, \mathbf{c}\left(z_{n}\right)\right)
$$

3. Stopping criterion.

(a) If $r^{\min } \leq \epsilon$, then stop; $v_{n}(x)$ is the approximate scattered field, and $A_{n}\left(\alpha^{\prime}, \alpha\right)$ is the approximate scattering amplitude.

(b) If $r^{\min }>\epsilon$, and $n<N_{\max }$, then repeat the iterative step (2).

(c) If $r^{\min }>\epsilon$, and $n=N_{\max }$, then the procedure failed.

\section{Numerical experiments for random multi-point MRC}

In this section we describe numerical results obtained by the Random Multi-point MRC method for 2D and $3 \mathrm{D}$ obstacles. We also compare the 2D results to the ones obtained by the Multiple-point MRC described above, and introduced in [13]. The results of [13] show a favorable comparison of the Multi-point MRC method with the Boundary Integral Equation Method. Further improvements are attained with the Random Multi-point MRC method, for which the Multi-point MRC is just the first iteration.

Note that in a 2D case instead of (3.1) one has

$$
\psi_{l}\left(x, x_{j}\right)=H_{l}^{(1)}\left(k\left|x-x_{j}\right|\right) \mathrm{e}^{\mathrm{i} l \theta_{j}},
$$

where $\left(x-x_{j}\right) /\left|x-x_{j}\right|=\mathrm{e}^{\mathrm{i} \theta_{j}}$.

For a numerical implementation choose $M$ nodes $\left\{t_{m}\right\}$ on the surface $S$ of the obstacle $D$. After the interior points $x_{j}, j=1,2, \ldots, J$ are chosen, form $N$ vectors

$$
\mathbf{a}^{(n)}=\left\{\psi_{l}\left(t_{m}, x_{j}\right)\right\}_{m=1}^{M},
$$


$n=1,2, \ldots, N$ of length $M$. Note that $N=(2 L+1) J$ for a 2D case, and $N=(L+1)^{2} J$ for a 3D case. It is convenient to normalize the norm in $\mathbb{R}^{M}$ by

$$
\|\mathbf{b}\|^{2}=\frac{1}{M} \sum_{m=1}^{M}\left|b_{m}\right|^{2}, \quad \mathbf{b}=\left(b_{1}, b_{2}, \ldots, b_{M}\right) .
$$

Then $\left\|u_{0}\right\|=1$.

Now let $\mathbf{b}=\left\{g\left(t_{m}\right)\right\}_{m=1}^{M}$, in the Random Multi-point MRC (see Section 3), and minimize

$$
\Phi(\mathbf{c})=\|\mathbf{b}+A \mathbf{c}\|,
$$

for $\mathbf{c} \in \mathbb{C}^{N}$, where $A$ is the matrix containing vectors $\mathbf{a}^{(n)}, n=1,2, \ldots, N$ as its columns.

The Singular Value Decomposition (SVD) method (see e.g. [24]) is used to minimize (4.1). According to the SVD, the matrix $A$ is represented as

$$
A=U W V^{*},
$$

where the $M \times M$ matrix $U$ has orthonormal columns $\mathbf{u}^{(n)}, n=1, \ldots, M$, the square $N \times N$ matrix $V$ has orthonormal columns $\mathbf{v}^{(n)}, n=1, \ldots, N$, and the $M \times N$ matrix $W=\left(w_{n}\right)_{n=1}^{N}$ is composed of the (nonnegative) singular values of $A, n=1, \ldots, r, r:=\operatorname{rank}(A)$ on the diagonal and zeros elsewhere.

Let $P \subset\{1,2, \ldots, N\}$ be defined by

$$
P=\left\{n: w_{n} \geq w_{\min }\right\}
$$

for some positive constant $w_{\min }$.

Compute the normalized residual

$$
r^{\min }=\frac{1}{\sqrt{M}} \sqrt{\|\mathbf{b}\|^{2}-\sum_{n \in P}\left|\left\langle\mathbf{u}^{(n)}, \mathbf{b}\right\rangle\right|^{2}} .
$$

The minimizer $\mathbf{c}$ is given by

$$
\mathbf{c}=\sum_{n \in P} \frac{1}{w_{n}}\left\langle\mathbf{u}^{(n)}, \mathbf{b}\right\rangle \mathbf{v}^{(n)} .
$$

Small singular values $w_{n}<w_{\min }$ of the matrix $A$ are used to identify and delete linearly dependent or almost linearly dependent combinations of vectors $\mathbf{a}^{(n)}$. This spectral cut-off makes the minimization process stable, see details in [13].

Let $r^{\min }$ be the residual, i.e. the minimal value of $\Phi(\mathbf{c})$ attained after $N_{\max }$ iterations of the Random Multi-point MRC method (or when it is stopped). For a comparison, let $r_{\text {old }}^{\min }$ be the residual obtained in [13] by the Multi-point MRC.

We have conducted 2D numerical experiments for four obstacles: two ellipses of different eccentricity, a kite, and a triangle. The $M=720$ nodes $t_{m}$ were uniformly distributed on the interval $[0,2 \pi]$, used to parametrize the boundary $S$. Each case was tested for wave numbers $k=1.0$ and $k=5.0$. Each obstacle was subjected to incident waves corresponding to $\alpha=(1.0,0.0)$ and $\alpha=(0.0,1.0)$.

The results for the Random Multi-point MRC with $J=1$ are shown in Table 1 , in the last column $r^{\min }$. In every experiment the target residual $\epsilon=0.0001$ was obtained in under 6000 iterations, in about 2 min run time on a 2.8 MHz PC.

In [13], we have conducted numerical experiments for the same four 2D obstacles by a Multi-point MRC, as described in the beginning of this section. The interior points $x_{j}$ were chosen differently in each experiment. Their choice is indicated in the description of each 2D experiment. The column $J$ shows the number of these interior points. Values $L=5$ and $M=720$ were used in all the experiments. These results are shown in Table 1, column $r_{\text {old }}^{\min }$.

Thus, the Random Multi-point MRC method achieved a significant improvement over the Multi-point MRC.

Experiment 2D-I. The boundary $S$ is an ellipse described by

$$
\mathbf{r}(t)=(2.0 \cos t, \sin t), \quad 0 \leq t<2 \pi .
$$


Table 1

Normalized residuals attained in the numerical experiments for $2 \mathrm{D}$ obstacles by Random Multi-point MRC, $\left\|\mathbf{u}_{0}\right\|=1$

\begin{tabular}{|c|c|c|c|c|c|}
\hline Experiment & $J$ & $k$ & $\alpha$ & $r_{\text {old }}^{\min }$ & $r^{\min }$ \\
\hline I & 4 & 1.0 & $(1.0,0.0)$ & 0.000201 & 0.0001 \\
\hline \multirow[t]{3}{*}{ Ellipse } & 4 & 1.0 & $(0.0,1.0)$ & 0.000357 & 0.0001 \\
\hline & 4 & 5.0 & $(1.0,0.0)$ & 0.001309 & 0.0001 \\
\hline & 4 & 5.0 & $(0.0,1.0)$ & 0.007228 & 0.0001 \\
\hline II & 16 & 1.0 & $(1.0,0.0)$ & 0.003555 & 0.0001 \\
\hline \multirow[t]{3}{*}{ Kite } & 16 & 1.0 & $(0.0,1.0)$ & 0.002169 & 0.0001 \\
\hline & 16 & 5.0 & $(1.0,0.0)$ & 0.009673 & 0.0001 \\
\hline & 16 & 5.0 & $(0.0,1.0)$ & 0.007291 & 0.0001 \\
\hline III & 16 & 1.0 & $(1.0,0.0)$ & 0.008281 & 0.0001 \\
\hline \multirow[t]{3}{*}{ Triangle } & 16 & 1.0 & $(0.0,1.0)$ & 0.007523 & 0.0001 \\
\hline & 16 & 5.0 & $(1.0,0.0)$ & 0.021571 & 0.0001 \\
\hline & 16 & 5.0 & $(0.0,1.0)$ & 0.024360 & 0.0001 \\
\hline IV & 32 & 1.0 & $(1.0,0.0)$ & 0.006610 & 0.0001 \\
\hline \multirow[t]{3}{*}{ Ellipse } & 32 & 1.0 & $(0.0,1.0)$ & 0.006785 & 0.0001 \\
\hline & 32 & 5.0 & $(1.0,0.0)$ & 0.034027 & 0.0001 \\
\hline & 32 & 5.0 & $(0.0,1.0)$ & 0.040129 & 0.0001 \\
\hline
\end{tabular}

Table 2

Normalized residuals attained in the numerical experiments for $3 \mathrm{D}$ obstacles by Random Multi-point MRC, $\left\|\mathbf{u}_{0}\right\|=1$

\begin{tabular}{|c|c|c|c|c|c|}
\hline Experiment & $k$ & $\alpha_{i}$ & $r^{\min }$ & $N_{\text {iter }}$ & Run time \\
\hline I & 1.0 & & 0.0002 & 1 & $1 \mathrm{~s}$ \\
\hline Sphere & 5.0 & & 0.001 & 700 & $7 \mathrm{~min}$ \\
\hline II & 1.0 & (1) & 0.001 & 800 & $16 \mathrm{~min}$ \\
\hline \multirow[t]{3}{*}{ Cube } & 1.0 & (2) & 0.001 & 200 & $4 \min$ \\
\hline & 5.0 & (1) & 0.0035 & 2000 & $40 \mathrm{~min}$ \\
\hline & 5.0 & (2) & 0.002 & 2000 & $40 \mathrm{~min}$ \\
\hline III & 1.0 & (1) & 0.001 & 3600 & $37 \mathrm{~min}$ \\
\hline \multirow[t]{3}{*}{ Ellipsoid } & 1.0 & (2) & 0.001 & 3000 & $31 \mathrm{~min}$ \\
\hline & 5.0 & (1) & 0.0026 & 5000 & $53 \mathrm{~min}$ \\
\hline & 5.0 & (2) & 0.001 & 5000 & $53 \mathrm{~min}$ \\
\hline
\end{tabular}

The Multi-point MRC used $J=4$ interior points $x_{j}=0.7 \mathbf{r}\left(\frac{\pi(j-1)}{2}\right), j=1, \ldots, 4$. The run time was $2 \mathrm{~s}$.

Experiment 2D-II. The kite-shaped boundary $S$ (see [9], Section 3.5) is described by

$$
\mathbf{r}(t)=(-0.65+\cos t+0.65 \cos 2 t, 1.5 \sin t), \quad 0 \leq t<2 \pi .
$$

The Multi-point MRC used $J=16$ interior points $x_{j}=0.9 \mathbf{r}\left(\frac{\pi(j-1)}{8}\right), j=1, \ldots, 16$. The run time was $33 \mathrm{~s}$.

Experiment 2D-III. The boundary $S$ is the triangle with vertices at $(-1.0,0.0)$ and $(1.0, \pm 1.0)$. The Multi-point MRC used the interior points $x_{j}=0.9 \mathbf{r}\left(\frac{\pi(j-1)}{8}\right), j=1, \ldots, 16$. The run time was about $30 \mathrm{~s}$.

Experiment 2D-IV. The boundary $S$ is an ellipse described by

$$
\mathbf{r}(t)=(0.1 \cos t, \sin t), \quad 0 \leq t<2 \pi .
$$

The Multi-point MRC used $J=32$ interior points $x_{j}=0.95 \mathbf{r}\left(\frac{\pi(j-1)}{16}\right), j=1, \ldots, 32$. The run time was about $140 \mathrm{~s}$.

The 3D numerical experiments were conducted for 3 obstacles: a sphere, a cube, and an ellipsoid. We used the Random Multi-point MRC with $L=0, w_{\min }=10^{-12}$, and $J=80$. The number $M$ of the points on the boundary $S$ is indicated in the description of the obstacles. The scattered field for each obstacle was computed for two incoming directions $\alpha_{i}=(\theta, \phi), i=1,2$, where $\phi$ was the polar angle. The first unit vector $\alpha_{1}$ is denoted by (1) in Table 2 , $\alpha_{1}=(0.0, \pi / 2)$. The second one is denoted by $(2), \alpha_{2}=(\pi / 2, \pi / 4)$. A typical number of iterations $N_{\text {iter }}$ and the 
Table 3

Normalized residuals attained in the numerical experiments for $2 \mathrm{D}$ obstacles by the Optimal Choice MRC, $\left\|\mathbf{u}_{0}\right\|=1$

\begin{tabular}{llllll}
\hline Experiment & $k$ & $\alpha$ & $N_{\text {iter }}$ & $r^{\min }$ & (MRC - BIEM)/BIEM \\
\hline I & 1.0 & $(1.0,0.0)$ & 20 & 0.0010 & 0.0001 \\
Ellipse & 1.0 & $(0.0,1.0)$ & 20 & 0.0018 & 0.0001 \\
& 5.0 & $(1.0,0.0)$ & 53 & 0.0010 & 0.0001 \\
II & 5.0 & $(0.0,1.0)$ & 45 & 0.0020 & 0.0001 \\
Kite & 1.0 & $(1.0,0.0)$ & 53 & 0.0020 & 0.0001 \\
& 1.0 & $(0.0,1.0)$ & 32 & 0.0020 & 0.0001 \\
& 5.0 & $(1.0,0.0)$ & 75 & 0.0020 & 0.0003 \\
III & 5.0 & $(1.0,1.0)$ & 68 & 0.0020 & 0.0001 \\
Triangle & 1.0 & $(0.0,1.0)$ & 55 & 0.0020 & 0.0017 \\
& 1.0 & $(1.0,0.0)$ & 48 & 0.0019 & 0.0020 \\
IV & $(0.0,1.0)$ & 72 & 80 & 0.0041 & 0.0008 \\
Ellipse & 5.0 & $(1.0,0.0)$ & 100 & 0.0027 & 0.0000 \\
& 5.0 & $(0.0,1.0)$ & 100 & 0.0058 & 0.0004 \\
V & 1.0 & $(1.0,0.0)$ & 100 & 0.0037 & 0.0012 \\
Circle & 1.0 & $(0.0,1.0)$ & 100 & 0.0000 & 0.0001 \\
\hline & 5.0 & $(1.0,0.0)$ & 21 & 0.0020 & 0.0001
\end{tabular}

run time on a 2.8 MHz PC are also shown in Table 2. For example, in experiment I with $k=5.0$ it took about 700 iterations of the Random Multi-point MRC method to achieve the target residual $r^{\mathrm{min}}=0.001$ in $7 \mathrm{~min}$.

Experiment 3D-I. The boundary $S$ is the sphere of radius 1, with $M=450$.

Experiment 3D-II. The boundary $S$ is the surface of the cube $[-1,1]^{3}$ with $M=1350$.

Experiment 3D-III. The boundary $S$ is the surface of the ellipsoid $x^{2} / 16+y^{2}+z^{2}=1$ with $M=450$.

In the last experiment the run time could be reduced by taking a smaller value for $J$. For example, the choice of $J=8$ reduced the running time to about $6-10 \mathrm{~min}$.

Numerical experiments show that the minimization results depend on the choice of such parameters as $J, w_{\min }$, and $L$.

\section{Numerical experiments for optimal choice MRC}

In this section we describe numerical results obtained by the MRC method with the optimal choice of sources for 2D and 3D obstacles. The notations are kept the same as in the previous section for the Random Multi-point MRC. As there, one has to minimize

$$
\Phi(z, \mathbf{c})=\|\mathbf{b}+A \mathbf{c}\|,
$$

in every iterative step, but, in addition, the residual is minimized with respect to the interior point $z \in D$.

There is a variety of methods to minimize $\Phi(z, \mathbf{c}(z))$, since after the minimization in the coefficients $\mathbf{c}(z)$ by the SVD it is just a 2D or 3D minimization in the region $D$. Our choice was the Powell's method which imitates the conjugate gradients approach, but does not require analytical expressions for the gradient. The Brent method was used for a line minimization, see $[24,16]$ for details. The Powell's algorithm is also described below in Section 10.

In addition to the four obstacles considered for the Random Multi-point MRC, the circle $|r|=1$ was tested to check if the Optimal point MRC was able to find the scattered field just after one iteration, since, in this case, the optimal point was in the origin. The result is in Table 3, experiment number $\mathrm{V}$. The column $N_{\text {iter }}$ shows the number of iterations (number of source points) at the end of the iterative process. The process was stopped after the algorithm reached the sought tolerance $\epsilon=0.002$, or $N_{\max }=100$. Values $L=5$ and $M=720$ were used in all 2D experiments.

The last column (MRC - BIEM)/BIEM shows the discrepancy in the scattering amplitude computed by the MRC and BIEM methods. The values shown are the $L_{2}$ norms of the difference of the scattering amplitude obtained by MRC and BIEM, over the $L_{2}$ norm of the scattering amplitude obtained by BIEM. We followed [9] for the BIEM 
Table 4

Normalized residuals attained in the numerical experiments for $3 \mathrm{D}$ obstacles by the Optimal Choice MRC, $\left\|\mathbf{u}_{0}\right\|=1$

\begin{tabular}{lllll}
\hline Experiment & $k$ & $\alpha_{i}$ & $N_{\text {iter }}$ & $r^{\min }$ \\
\hline I & 1.0 & & 1 & 0.0000 \\
Sphere & 5.0 & & 43 & 0.0019 \\
II & 1.0 & $(1)$ & 12 & 0.0019 \\
Cube & 1.0 & $(2)$ & 7 & 0.0019 \\
& 5.0 & $(1)$ & 35 & 0.0019 \\
III & 5.0 & $(2)$ & 12 & 0.0020 \\
Ellipsoid & 1.0 & $(1)$ & 35 & 0.0016 \\
& 1.0 & $(1)$ & 55 & 0.0020 \\
& 5.0 & $(2)$ & 67 & 0.0020 \\
\hline
\end{tabular}

implementation using 64 points on the boundary $S$ in every 2D experiment. No comparison is provided for a triangular obstacle, since it requires a complete rewriting of the BIEM code to accommodate the corner points. No such rewriting is required for the MRC method. Table 3 shows that for the value of tolerance $\epsilon=0.002$ the computed scattering amplitude is in an excellent agreement with the scattering amplitude computed using BIEM. Results for 3D obstacles are provided in Table 4.

Concerning the efficiency of the methods: for simple geometries the Multi-point MRC (see [13]) is the fastest, provided that the required accuracy can be achieved by a relatively small number $J$ of the interior points (sources) used simultaneously. This assures the resulting matrices being of a manageable size. Otherwise, one has to use Random, or Optimal choice MRC, which take a significantly longer time to run, but can accomplish the solution of scattering problems untractable by single-step methods, such as the Multi-point MRC or BIEM. While the precision of the Random-point MRC was higher in the conducted experiments, the optimally placed MRC method achieves an order of magnitude improvement in run time over it.

\section{MRC for static problems}

In this section we follow [35,34], Chapter 12. Consider a bounded domain $D \subset \mathbb{R}^{3}$ with a Lipschitz boundary $S$, $D \subset B_{R}:=\{x:|x| \leq R\}$. Denote the exterior domain by $D^{\prime}=\mathbb{R}^{3} \backslash D$. Let $S^{2}$ denote the unit sphere in $\mathbb{R}^{3}$. Consider the problem:

$$
\begin{aligned}
& \nabla^{2} v=0 \quad \text { in } D^{\prime}, v=f \text { on } S, \\
& v:=O\left(\frac{1}{r}\right), \quad r:=|x| \rightarrow \infty .
\end{aligned}
$$

Denote by $Y_{\ell}(\alpha), \alpha \in S^{2}$ the orthonormal spherical harmonics, $Y_{\ell}=Y_{\ell m},-\ell \leq m \leq \ell$, and let harmonic functions $H_{\ell}(x)$ be defined by

$$
H_{\ell}(x):=\frac{Y_{\ell}(\alpha)}{r^{\ell+1}}, \quad \ell \geq 0, \alpha:=\frac{x}{r} \in S^{2} .
$$

In the region $r>R$ the solution to (6.1) and (6.2) is:

$$
v(x)=\sum_{\ell=0}^{\infty} c_{\ell} H_{\ell}(x), \quad r>R .
$$

The summation in (6.3) and below includes summation with respect to $m,-\ell \leq m \leq \ell$, and $c_{\ell}=c_{\ell, m}$ are some coefficients determined by $f$.

The series (6.3) in general does not converge up to the boundary $S$. Our aim is to give a formulation of an analog of the Modified Rayleigh Conjecture (MRC) from [27], which can be used in numerical solution of the boundary-value problems. The authors hope that the MRC method for static problems can be used as a basis for an efficient numerical 
algorithm for solving boundary-value problems for Laplace equations in domains with complicated boundaries. In above sections such algorithms were developed on the basis of MRC for solving boundary-value problems for the Helmholtz equation. Although the boundary integral equation methods and finite elements methods are widely and successfully used for solving these problems, the method, based on MRC, proved to be competitive and often superior to the currently used methods.

We discuss the Dirichlet condition but a similar argument is applicable to the Neumann and Robin boundary conditions. Boundary-value problems and scattering problems in rough domains were studied in [28] and in [34], Chapter 9.

Let us present the basic results on which the MRC method is based.

Fix $\epsilon>0$, an arbitrary small number.

Lemma 6.1. There exist $L=L(\epsilon)$ and $c_{\ell}=c_{\ell}(\epsilon)$ such that

$$
\left\|\sum_{\ell=0}^{L(\epsilon)} c_{\ell}(\epsilon) H_{\ell}-f\right\|_{L^{2}(S)} \leq \epsilon .
$$

If (6.4) and the boundary condition (6.1) hold, then

$$
\left\|v_{\epsilon}-v\right\|_{L^{2}(S)} \leq \epsilon, \quad v_{\epsilon}:=\sum_{\ell=0}^{L(\epsilon)} c_{\ell}(\epsilon) H_{\ell} .
$$

Lemma 6.2. If (6.4) holds then

$$
\left\|v_{\epsilon}-v\right\|=O(\epsilon) \quad \epsilon \rightarrow 0
$$

where $\|\cdot\|:=\|\cdot\|_{H_{\mathrm{loc}}^{m}\left(D^{\prime}\right)}+\|\cdot\|_{L^{2}\left(D^{\prime} ;(1+|x|)^{-\gamma}\right)}, \gamma>1, m>0$ is an arbitrary integer, and $H^{m}$ is the Sobolev space.

In particular, (6.6) implies

$$
\left\|v_{\epsilon}-v\right\|_{L^{2}\left(S_{R}\right)}=O(\epsilon) \quad \epsilon \rightarrow 0 .
$$

Let us formulate an analog of the Modified Rayleigh Conjecture (MRC):

Theorem 6.3 (MRC). For an arbitrary small $\epsilon>0$ there exist $L(\epsilon)$ and $c_{\ell}(\epsilon), 0 \leq \ell \leq L(\epsilon)$, such that (6.4) hold.

Theorem 6.3 follows from Lemmas 6.1 and 6.2.

For the Neumann boundary condition one minimizes $\left\|\frac{\partial\left[\sum_{\ell=0}^{L} c_{\ell} \psi_{\ell}\right]}{\partial N}-f\right\|_{L^{2}(S)}$ with respect to $c_{\ell}$. Analogs of Lemmas 6.1 and 6.2 are valid and their proofs are essentially the same.

If the boundary data $f \in C(S)$, then one can use $C(S)$-norm in (6.4)-(6.7), and an analog of Theorem 6.3 then follows immediately from the maximum principle.

To solve problem (6.1) and (6.2) using MRC, fix a small $\epsilon>0$ and find $L(\epsilon)$ and $c_{\ell}(\epsilon)$ such that (6.4) holds. This is possible by Lemma 6.1 and can be done numerically by minimizing $\left\|\sum_{0}^{L} c_{\ell} H_{\ell}-f\right\|_{L^{2}(S)}:=\phi\left(c_{1}, \ldots, c_{L}\right)$. If the minimum of $\phi$ is larger than $\epsilon$, then increase $L$ and repeat the minimization. Lemma 6.1 guarantees the existence of such $L$ and $c_{\ell}$ that the minimum is less than $\epsilon$. Choose the smallest $L$ for which this happens and define $v_{\epsilon}:=\sum_{\ell=0}^{L} c_{\ell} H_{\ell}$. Then, by Lemma 6.2, $v_{\epsilon}$ is the approximate solution to problem (6.1) and (6.2) with the accuracy $O(\epsilon)$ in the norm $\|\cdot\|$.

Proof of Lemma 6.1. We start with the claim:

Claim. The restrictions of harmonic functions $H_{\ell}$ on $S$ form a total set in $L^{2}(S)$.

Lemma 6.1 follows from this claim. Let us prove the claim. Assume the contrary. Then there is a function $g \neq 0$ such that $\int_{S} g(s) h_{\ell}(s) \mathrm{d} s=0 \forall \ell \geq 0$. This implies $V(x):=\int_{S} g(s)|x-s|^{-1} \mathrm{~d} s=0 \forall x \in D^{\prime}$. Thus $V=0$ on $S$, and since $\Delta V=0$ in $D$, one concludes that $V=0$ in $D$. Thus $g=0$ by the jump formula for the normal derivatives of the simple layer potential $V$. This contradiction proves the claim. Lemma 6.1 is proved. 
Proof of Lemma 6.2. By Green's formula one has

$$
w_{\epsilon}(x)=\int_{S} w_{\epsilon}(s) G_{N}(x, s) \mathrm{d} s, \quad\left\|w_{\epsilon}\right\|_{L^{2}(S)}<\epsilon, w_{\epsilon}:=v_{\epsilon}-v .
$$

Here $N$ is the unit normal to $S$, pointing into $D^{\prime}$, and $G$ is the Dirichlet Green's function of the Laplacian in $D^{\prime}$ :

$$
\begin{aligned}
& \nabla^{2} G=-\delta(x-y) \quad \text { in } D^{\prime}, G=0 \text { on } S, \\
& G=O\left(\frac{1}{r}\right), \quad r \rightarrow \infty .
\end{aligned}
$$

From (6.8) one gets (6.7) and (6.6) with $H_{\mathrm{loc}}^{m}\left(D^{\prime}\right)$-norm immediately by the Cauchy inequality. Estimate (6.6) in the region $B_{R}^{\prime}:=\mathbb{R}^{3} \backslash B_{R}$ follows from the estimate

$$
\left|G_{N}(x, s)\right| \leq \frac{c}{1+|x|}, \quad|x| \geq R .
$$

In the region $B_{R} \backslash D$ estimate (6.6) follows from local elliptic estimates for $w_{\epsilon}:=v_{\epsilon}-v$, which imply that

$$
\left\|w_{\epsilon}\right\|_{L^{2}\left(B_{R} \backslash D\right)} \leq c \epsilon .
$$

Let us recall the elliptic estimate we have used. Let $D_{R}^{\prime}:=B_{R} \backslash D$ and $S_{R}$ be the boundary of $B_{R}$. Recall the elliptic estimate for the solution to homogeneous Laplace equation in $D_{R}^{\prime}$ (see [20], p. 189):

$$
\left\|w_{\epsilon}\right\|_{H^{0.5}\left(D_{R}^{\prime}\right)} \leq c\left[\left\|w_{\epsilon}\right\|_{L^{2}\left(S_{R}\right)}+\left\|w_{\epsilon}\right\|_{L^{2}(S)}\right] .
$$

The estimates $\left\|w_{\epsilon}\right\|_{L^{2}\left(S_{R}\right)}=O(\epsilon),\left\|w_{\epsilon}\right\|_{L^{2}(S)}=O(\epsilon)$, and (6.13) yield (6.6). Lemma 6.2 is proved.

\section{MRC for scattering by periodic structures}

Determination of fields scattered by periodic structures is of a great importance in modern diffractive optics, and there is a vast literature on both the direct and inverse problems of this type, see, for example [23]. Still, an efficient computation of such fields presents certain difficulties. In the next sections we present some theoretical background, a modification of the MRC method, and numerical results for such a scattering, see [29].

For simplicity we consider a 2-D setting, but our arguments can be as easily applied to $n$-dimensional problems, $n \geq 2$. Let $f: \mathbb{R} \rightarrow \mathbb{R}, f(x+L)=f(x)$ be an $L$-periodic Lipschitz continuous function, and let $D$ be the domain

$$
D=\{(x, y): y \geq f(x), x \in \mathbb{R}\} .
$$

Without loss of generality we assume that $f \geq 0$. If it is not, one can choose the origin so that this assumption is satisfied, because $M:=\sup _{0 \leq x \leq L}|f(x)|<\infty$.

Let $\mathbf{x}=(x, y)$ and $u(\mathbf{x})$ be the total field satisfying

$$
\begin{aligned}
& \left(\Delta+k^{2}\right) u=0, \quad \mathbf{x} \in D, \quad k=\text { const }>0 \\
& u=0 \quad \text { on } S:=\partial D, \\
& u=u_{0}+v, \quad u_{0}:=\mathrm{e}^{\mathrm{i} k \alpha \cdot \mathbf{x}},
\end{aligned}
$$

where the unit vector $\alpha=(\cos \theta,-\sin \theta), 0<\theta<\pi / 2$, and $v(\mathbf{x})$ is the scattered field, whose asymptotic behavior as $y \rightarrow \infty$ will be specified below, and

$$
u(x+L, y)=v u(x, y), \quad u_{x}(x+L, y)=v u_{x}(x, y) \quad \text { in } D, v:=\mathrm{e}^{\mathrm{i} k L \cos \theta} .
$$

Conditions (7.4) are the $q p$ (quasiperiodicity) conditions. To find the proper radiation condition for the scattered field $v(\mathbf{x})$ consider the spectral problem

$$
\begin{aligned}
& \varphi^{\prime \prime}+\ell^{2} \varphi=0, \quad 0<x<L, \\
& \varphi(L)=v \varphi(0), \quad \varphi^{\prime}(L)=v \varphi^{\prime}(0)
\end{aligned}
$$


arising from the separation of variables in (7.1)-(7.4). This problem has a discrete spectrum, and its eigenfunctions form a basis in $L^{2}(0, L)$. One can show that the corresponding eigenfunctions are $\mathrm{e}^{\mathrm{i} \ell_{j}^{+} x}$ and $e^{-\mathrm{i} \ell_{j}^{-} x}$ with

$$
\ell_{j}^{+}=k \cos \theta+\frac{2 \pi j}{L}, \quad \text { or } \quad \ell_{j}^{-}=-k \cos \theta+\frac{2 \pi j}{L}, \quad j=0, \pm 1, \pm 2, \ldots
$$

We will use the system $\mathrm{e}^{\mathrm{i} \ell_{j}^{+} x}$, which forms an orthogonal basis in $L^{2}(0, L)$. One has:

$$
\int_{0}^{L} \mathrm{e}^{\mathrm{i} \ell_{j}^{+} x} \mathrm{e}^{-\mathrm{i} \ell_{m}^{+} x} \mathrm{~d} x=\int_{0}^{L} \mathrm{e}^{\frac{2 \pi \mathrm{i}}{L}(j-m)} \mathrm{d} x=0, \quad j \neq m .
$$

The normalized eigenfunctions are

$$
\varphi_{j}(x)=\frac{\mathrm{e}^{\mathrm{i} \ell_{j}^{+} x}}{\sqrt{L}}, \quad j=0, \pm 1, \pm 2, \ldots
$$

These functions form an orthonormal basis of $L^{2}(0, L)$.

Let us look for $v(\mathbf{x})=v(x, y)$ of the form

$$
v(x, y)=\sum_{j=-\infty}^{\infty} c_{j} v_{j}(y) \varphi_{j}(x), \quad y>M, c_{j}=\text { const. }
$$

For $y>M$, (7.1) implies

$$
v_{j}^{\prime \prime}+\left(k^{2}-\ell_{j}^{2}\right) v_{j}=0 .
$$

Let us assume that $\ell_{j}^{2} \neq k^{2}$ for all $j$. Then

$$
v_{j}(y)=\mathrm{e}^{\mathrm{i} \mu_{j} y},
$$

where, for finitely many $j$, the set of which is denoted by $J$, one has:

$$
\mu_{j}=\left(k^{2}-\ell_{j}^{2}\right)^{1 / 2}>0, \quad \text { if } \ell_{j}^{2}<k^{2}, j \in J,
$$

and

$$
\mu_{j}=\mathrm{i}\left(\ell_{j}^{2}-k^{2}\right)^{1 / 2}, \quad \text { if } \ell_{j}^{2}>k^{2}, j \notin J .
$$

The radiation condition at infinity requires that the scattered field $v(x, y)$ be representable in the form (7.7) with $v_{j}(y)$ defined by (7.9)-(7.11).

The Periodic Scattering Problem consists of finding the solution to (7.1)-(7.4) satisfying the radiation condition (7.7) and (7.9)-(7.11).

The existence and uniqueness for such a scattering problem is established in [29]. In [1] the scattering by a periodic structure was considered earlier, and was based on a uniqueness theorem from [12]. There are many papers on scattering by periodic structures, of which we mention a few $[1,2,4,5,8,17,18,22,21,23,36]$. The Rayleigh conjecture is discussed in several of the above papers. It was shown (see e.g. [23,3]) that this conjecture is incorrect, in general. As we have already discussed in the previous sections, the modified Rayleigh conjecture is a theorem proved in [27] for scattering by bounded obstacles.

The main ingredient in the solution is an analog to the half-space Dirichlet Green's function. The function $g=g(\mathbf{x}, \xi, k)$ can be constructed analytically $\left(\mathbf{x}=\left(x_{1}, x_{2}\right), \xi=\left(\xi_{1}, \xi_{2}\right)\right)$ :

$$
\begin{aligned}
& g(\mathbf{x}, \xi)=\sum_{j} \varphi_{j}\left(x_{1}\right) \overline{\varphi_{j}\left(\xi_{1}\right)} g_{j}\left(x_{2}, \xi_{2}, k\right), \\
& g_{j}:=g_{j}\left(x_{2}, \xi_{2}, k\right)= \begin{cases}v_{j}\left(x_{2}\right) \psi_{j}\left(\xi_{2}\right), & x_{2}>\xi_{2} \\
v_{j}\left(\xi_{2}\right) \psi_{j}\left(x_{2}\right), & x_{2}<\xi_{2}\end{cases} \\
& \psi_{j}=\left(\mu_{j}\right)^{-1} \mathrm{e}^{\mathrm{i} \mu_{j} b} \sin \left[\mu_{j}\left(\xi_{2}+b\right)\right], \quad \mu_{j}=\left[k^{2}-\lambda_{j}^{2}\right]^{1 / 2}, \quad v_{j}\left(x_{2}\right)=\mathrm{e}^{\mathrm{i} \mu_{j} x_{2}},
\end{aligned}
$$


where

$$
\psi_{j}^{\prime \prime}+\left(k^{2}-\ell_{j}^{2}\right) \psi_{j}=0, \quad \psi_{j}(-b)=0, \quad W\left[v_{j}, \psi_{j}\right]=1, \quad \lambda_{j}=k \cos (\theta)+\frac{2 \pi j}{L},
$$

and $W[v, \psi]$ is the Wronskian.

The function $g$ is analytic with respect to $k$ on the complex plain with cuts along the rays $\lambda_{j}-\mathrm{i} \tau, 0 \leq \tau<\infty, j=$ $0, \pm 1, \pm 2, \ldots$, in particular, in the region $\Im k>0$, up to the real positive half-axis except for the set $\left\{\lambda_{j}\right\}_{j=0, \pm 1, \pm 2, \ldots}$.

Choose $b>0$ such that $k^{2}>0$ is not an eigenvalue of the problem:

$$
\begin{aligned}
& \left(\Delta+k^{2}\right) \psi=0, \quad \text { in } D_{-b}:=\{(x, y):-b \leq y \leq f(x), 0 \leq x \leq L\} . \\
& \left.\psi\right|_{y=-b}=0, \quad \psi_{N}=0 \quad \text { on } S, \\
& \psi(x+L, y)=v \psi(x, y), \quad \psi_{x}(x+L, y)=v \psi_{x}(x, y) .
\end{aligned}
$$

One has

$$
\begin{aligned}
& \left(\Delta+k^{2}\right) g=-\delta(\mathbf{x}-\xi), \quad \mathbf{x}=\left(x_{1}, x_{2}\right), \xi=\left(\xi_{1}, \xi_{2}\right), \\
& \mathbf{x} \in\{(x, y):-b<y<\infty, 0 \leq x \leq L\}, \\
& \left.g\right|_{y=-b}=0 .
\end{aligned}
$$

Rayleigh conjectured [36] ("Rayleigh hypothesis") that the series (7.7) converges up to the boundary $S_{L}$. This conjecture is wrong [23] for some $f(x)$. Since the Rayleigh hypothesis has been widely used for numerical solution of the scattering problem by physicists and engineers, and because these practitioners reported high instability of the numerical solution, and there are no error estimates, we propose a modification of the Rayleigh conjecture, which is a theorem. This MRC (Modified Rayleigh Conjecture) can be used for a numerical solution of the scattering problem, and it gives an error estimate for this solution. Our arguments are very similar to the ones in [27].

Rewrite the scattering problem (7.1)-(7.4) as

$$
\left(\Delta+k^{2}\right) v=0 \quad \text { in } D, v=-u_{0} \text { on } S_{L},
$$

where $v$ satisfies (7.4), and $v$ has representation (7.7), that is, $v$ is "outgoing", it satisfies the radiation condition. Fix an arbitrarily small $\epsilon>0$, and assume that

$$
\left\|u_{0}+\sum_{|j| \leq j(\epsilon)} c_{j}(\epsilon) v_{j}(y) \varphi_{j}(x)\right\| \leq \epsilon, \quad 0 \leq x \leq L, y=f(x),
$$

where $\|\cdot\|=\|\cdot\|_{L^{2}\left(S_{L}\right)}$.

Lemma 7.1. For any $\epsilon>0$, however small, and for any $u_{0} \in L^{2}\left(S_{L}\right)$, there exists $j(\epsilon)$ and $c_{j}(\epsilon)$ such that (7.18) holds.

Proof. Let us prove the completeness of the system $\left\{\varphi_{j}(x) v_{j}(f(x))\right\}_{j=0, \pm 1, \pm 2, \ldots}$ in $L^{2}\left(S_{L}\right)$. Assume that there is an $h \in L^{2}\left(S_{L}\right), h \not \equiv 0$ such that

$$
\int_{S_{L}} h \overline{\varphi_{j}(x)} v_{j}(f(x)) \mathrm{d} s=0
$$

for all $j$. From (7.19) one derives (cf. [28], p. 162-163)

$$
\psi(\mathbf{x}):=\int_{S_{L}} h g(\mathbf{x}, \xi) \mathrm{d} \xi=0, \quad \mathbf{x} \in D_{-b} .
$$

Thus $\psi=0$ in $D_{L}$, and $h=\psi_{N}^{+}-\psi_{N}^{-}=0$. Lemma 7.1 is proved.

Lemma 7.2. If (7.18) holds, then

$$
\left\|v(\mathbf{x})-\sum_{|j| \leq j(\epsilon)} c_{j}(\epsilon) v_{j}(y) \varphi_{j}(x)\right\| \leq c \epsilon, \quad \forall x, y \in D_{L}, 0 \leq x \leq L, y=f(x),
$$


where $c=$ const $>0$ does not depend on $\epsilon, x, y ; R>M$ is an arbitrary fixed number, and $\|w\|=$ $\sup _{\mathbf{x} \in D \backslash D_{L R}}|w(\mathbf{x})|+\|w\|_{H^{1 / 2}\left(D_{L R}\right)}$.

See [29] for the proof.

From Lemma 7.2 the basic result, Theorem 7.3, follows immediately:

Theorem 7.3 (MRC-Modified Rayleigh Conjecture). Fix $\epsilon>0$, however small, and choose a positive integer p. Find

$$
\min _{c_{j}}\left\|u_{0}+\sum_{|j| \leq p} c_{j} \varphi_{j}(x) v_{j}(y)\right\|:=m(p) .
$$

Let $\left\{c_{j}(p)\right\}$ be the minimizer of (7.21). If $m(p) \leq \epsilon$, then

$$
v(p)=\sum_{|j| \leq p} c_{j}(p) \varphi_{j}(x) v_{j}(y)
$$

satisfies the inequality

$$
\|v-v(p)\| \leq c \epsilon,
$$

where $c=$ const $>0$ does not depend on $\epsilon$. If $m(p)>\epsilon$, then there exists $j=j(\epsilon)>p$ such that $m(j(\epsilon))<\epsilon$. Denote $c_{j}(j(\epsilon)):=c_{j}(\epsilon)$ and $v(j(\epsilon)):=v_{\epsilon}$. Then

$$
\left\|v-v_{\epsilon}\right\| \leq c \epsilon .
$$

\section{Numerical solution of the periodic scattering problem}

According to the MRC method (Theorem 7.3), if the restriction of the incident field $-u_{0}(x, y)$ to $S_{L}$ is approximated as in (7.21), then the series (7.22) approximates the scattered field in the entire region above the profile $y=f(x)$. However, a numerical method that uses (7.21) does not produce satisfactory results as reported in [23] and elsewhere. Our own numerical experiments confirm this observation. A way to overcome this difficulty is to realize that the numerical approximation of the field $-\left.u_{0}\right|_{S_{L}}$ can be carried out by using outgoing solutions described below.

Let $\xi=\left(\xi_{1}, \xi_{2}\right) \in D_{-b}$, where $b>0$,

$$
D_{-b}:=\left\{\left(\xi_{1}, \xi_{2}\right):-b \leq \xi_{2} \leq f(x), 0 \leq \xi_{1} \leq L\right\},
$$

and $g(\mathbf{x}, \xi)$ be defined as in (7.12). Then $g(\mathbf{x}, \xi)$ is an outgoing solution satisfying $\Delta g+k^{2} g=0$ in $D_{L}$, according to (7.15).

To implement the MRC method numerically one proceeds as follows:

1. Choose the nodes $\mathbf{x}_{i}, i=1,2, \ldots, N$ on the profile $S_{L}$. These points are used to approximate $L^{2}$ norms on $S_{L}$.

2. Choose points $\xi^{(1)}, \xi^{(2)}, \ldots, \xi^{(M)}$ in $D_{-b}, M<N$.

3. Form the vectors $\mathbf{b}=\left(u_{0}\left(\mathbf{x}_{i}\right)\right)$, and $\mathbf{a}^{(m)}=\left(g\left(\mathbf{x}_{i}, \xi^{(m)}\right)\right), i=1,2, \ldots, N, m=1,2, \ldots, M$. Let $\mathbf{A}$ be the $N \times M$ matrix containing vectors $\mathbf{a}^{(m)}$ as its columns.

4. Find the Singular Value Decomposition of A. Use a predetermined $w_{\min }>0$ to eliminate its small singular values. Use the decomposition to compute

$$
r^{\min }=\min \left\{\|\mathbf{b}+\mathbf{A c}\|, \mathbf{c} \in \mathbb{C}^{M}\right\},
$$

where

$$
\|\mathbf{a}\|^{2}=\frac{1}{N} \sum_{i=1}^{N}\left|a_{i}\right|^{2} .
$$

5. Stopping criterion. Let $\epsilon>0$. 
Table 5

Residuals attained in the numerical experiments on MRC for periodic structures

\begin{tabular}{|c|c|c|}
\hline Profile & $\theta$ & $r^{\min }$ \\
\hline I & $\begin{array}{l}\pi / 4 \\
\pi / 3 \\
\pi / 2\end{array}$ & $\begin{array}{l}0.000424 \\
0.000407 \\
0.000371\end{array}$ \\
\hline II & $\begin{array}{l}\pi / 4 \\
\pi / 3 \\
\pi / 2\end{array}$ & $\begin{array}{l}0.001491 \\
0.001815 \\
0.002089\end{array}$ \\
\hline III & $\begin{array}{l}\pi / 4 \\
\pi / 3 \\
\pi / 2\end{array}$ & $\begin{array}{l}0.009623 \\
0.011903 \\
0.013828\end{array}$ \\
\hline IV & $\begin{array}{l}\pi / 4 \\
\pi / 3 \\
\pi / 2\end{array}$ & $\begin{array}{l}0.014398 \\
0.017648 \\
0.020451\end{array}$ \\
\hline
\end{tabular}

(a) If $r^{\min } \leq \epsilon$, then stop. Use the coefficients $\mathbf{c}=\left\{c_{1}, c_{2}, \ldots, c_{M}\right\}$ obtained in the above minimization step to compute the scattered field by

$$
v(x, y)=\sum_{m=1}^{M} c_{m} g\left(x, y, \xi^{(m)}\right) .
$$

(b) If $r^{\text {min }}>\epsilon$, then increase $N, M$ by the order of 2, readjust the location of points $\xi^{(m)} \in D_{-b}$ as needed, and repeat the procedure.

We conducted numerical experiments for four different profiles. In each case we used $L=\pi, k=1.0$ and three values for the angle $\theta$. Table 5 shows the resulting residuals $r^{\min }$. Note that $\|\mathbf{b}\|=1$. Thus, in all the considered cases, the MRC method achieved $0.04 \%-2 \%$ accuracy of the approximation. Other parameters used in the experiments were chosen as follows: $N=256, M=64, w_{\min }=10^{-8}, b=1.2$. The value of $b>0$, used in the definition of $g$, was chosen experimentally, but the dependency of $r^{\mathrm{min}}$ on $b$ was slight. The Singular Value Decomposition (SVD) is used in Step 4 since the vectors $\mathbf{a}^{(m)}, m=1,2, \ldots, M$ may be nearly linearly dependent, which leads to an instability in the determination of the minimizer c. According to the SVD method this instability is eliminated by cutting off small singular values of the matrix A, see e.g. [24] for details. The cut-off value $w_{\min }>0$ was chosen experimentally. We used the truncated series (7.12) with $|j| \leq 120$ to compute functions $g(x, y, \xi)$. A typical run time on a $333 \mathrm{MHz} \mathrm{PC}$ was about $40 \mathrm{~s}$ for each experiment.

The following is a description of the profiles $y=f(x)$, the nodes $\mathbf{x}_{i} \in S_{L}$, and the poles $\xi^{(m)} \in D_{-b}$ used in the computation of $g\left(\mathbf{x}_{i}, \xi^{(m)}\right)$ in Step 3. For example, in profile I the $x$-coordinates of the $N$ nodes $\mathbf{x}_{i} \in S_{L}$ are uniformly distributed on the interval $0 \leq x \leq L$. The poles $\xi^{(m)} \in D_{-b}$ were chosen as follows: every fourth node $\mathbf{x}_{i}$ was moved by a fixed amount -0.1 parallel to the $y$ axis, so it would be within the region $D_{-b}$. The location of the poles was chosen experimentally to give the smallest value of the residual $r^{\min }$.

Profile I. $f(x)=\sin (2 x)$ for $0 \leq x \leq L, t_{i}=i L / N, \mathbf{x}_{i}=\left(t_{i}, f\left(t_{i}\right)\right), i=1,2, \ldots, N, \xi^{(m)}=\left(x_{4 m}, y_{4 m}-\right.$ $0.1), m=1,2, \ldots, M$.

Profile II. $f(x)=\sin (0.2 x)$ for $0 \leq x \leq L, t_{i}=i L / N, \mathbf{x}_{i}=\left(t_{i}, f\left(t_{i}\right)\right), i=1,2, \ldots, N, \xi^{(m)}=$ $\left(x_{4 m}, y_{4 m}-0.1\right), m=1,2, \ldots, M$.

Profile III. $f(x)=x$ for $0 \leq x \leq L / 2, f(x)=L-x$ for $L / 2 \leq x \leq L, t_{i}=i L / N, \mathbf{x}_{i}=\left(t_{i}, f\left(t_{i}\right)\right), i=$ $1,2, \ldots, N, \xi^{(m)}=\left(x_{4 m}, y_{4 m}-0.1\right), m=1,2, \ldots, M$.

Profile IV. $f(x)=x$ for $0 \leq x \leq L, t_{i}=2 i L / N, \mathbf{x}_{i}=\left(t_{i}, f\left(t_{i}\right)\right), i=1, \ldots, N / 2, \mathbf{x}_{i}=(L, f(2(i-$ $N / 2) L / N)), i=N / 2+1, \ldots, N, \xi^{(m)}=\left(x_{4 m}-0.03, y_{4 m}-0.05\right), m=1,2, \ldots, M$. In this profile $N / 2$ nodes $\mathbf{x}_{i}$ are uniformly distributed on its slant part, and $N / 2$ nodes are uniformly distributed on its vertical portion $x=L$.

The experiments show that the MRC method provides a competitive alternative to other methods for the computation of fields scattered from periodic structures. It is fast and inexpensive. The results depend on the number of the internal points $\xi^{(m)}$ and on their location. 


\section{Inverse scattering methods based on the MRC}

Suppose that an approximate location of the obstacle $D$ is obtained a numerical inversion method, such as the Support Function Method (SFM), see [15,30]. Then one can try to use the MRC method to improve the location of the boundary, see [27]. Such methods are under development by the authors, and they are going to be discussed elsewhere. Nevertheless, the MRC provides a tool for an easy construction of various examples illustrating the severe ill-posedness of the Inverse Scattering problem, which can be used for the algorithm's testing.

Here we present one such example. Let the obstacle $D$ be the unit circle $\left\{\mathbf{x} \in \mathbb{R}^{2}:|\mathbf{x}| \leq 1\right\}$. If the incident field is $u_{0}(x)=\mathrm{e}^{\mathrm{i} k x \cdot \alpha}$, then the scattered field $v(x)=-u_{0}(x)$ for $x \in S=\partial D$, and its scattering amplitude is

$$
A\left(\alpha^{\prime}, \alpha\right)=-\sqrt{\frac{2}{\pi k}} \mathrm{e}^{-\mathrm{i} \frac{\pi}{4}} \sum_{l=-\infty}^{\infty} \frac{J_{l}(k a)}{H_{l}^{(1)}(k a)} \mathrm{e}^{\mathrm{i} l(\theta-\beta)},
$$

where $\alpha^{\prime}=\mathbf{x} /|\mathbf{x}|=\mathrm{e}^{\mathrm{i} \theta}$, and $\alpha=\mathrm{e}^{\mathrm{i} \beta}$.

Let $x_{1} \in \mathbb{R}^{2}$. Fix an integer $L>0$, and let $\mathbf{c} \in \mathbb{C}^{2 L+1}$. Form the radiating solution

$$
v_{c}(x)=\sum_{l=-L}^{L} c_{l} H_{l}^{(1)}\left(k\left|x-x_{1}\right|\right) \mathrm{e}^{\mathrm{i} l \theta_{1}},
$$

where $\left(x-x_{1}\right) /\left|x-x_{1}\right|=\mathrm{e}^{\mathrm{i} \theta_{1}}$. Then its far field pattern is

$$
A_{v_{c}}\left(\alpha^{\prime}\right)=\sqrt{\frac{2}{\pi k}} \mathrm{e}^{-\mathrm{i} \frac{\pi}{4}}\left(\mathrm{e}^{-\mathrm{i} k \alpha^{\prime} \cdot x_{1}} \sum_{l=-L}^{L} c_{l}(-\mathrm{i})^{l} \mathrm{e}^{\mathrm{i} l \theta}\right),
$$

where $\alpha^{\prime}=x /|x|=\mathrm{e}^{\mathrm{i} \theta}$.

Fix an $\alpha \in S^{1}$, and let

$$
r^{\min }=\min \left\{\left\|A_{v_{c}}\left(\alpha^{\prime}\right)-A\left(\alpha^{\prime}, \alpha\right)\right\|: \mathbf{c} \in \mathbb{C}^{2 L+1}\right\} .
$$

We conducted the minimization by the Singular Value Decomposition Method with the following values of the parameters: $k=1.0, L=5, \alpha=(1.0,0.0)$, and $x_{1}=(0.8,0.0)$. The $L^{2}$ norm in (9.4) was computed over $M=120$ directions $\alpha_{m}^{\prime}$ uniformly distributed in the unit circle $S^{1}$, and then normalized by $\sqrt{M}$, so that the identity function would have the norm equal to 1 . The resulting value of the residual $r^{\min }=0.00009776$ indicates that the far field $A\left(\alpha^{\prime}, \alpha\right)$ was practically perfectly fit by the radiating solution of the form (9.2). However, as the Table 6 shows, the restrictions of the exact scattered field $v$, and the fitted field $v_{c}$ to the boundary $S$ of the obstacle $D$ are vastly different. The columns in Table 6 correspond to the real and the imaginary parts of the scattered fields, and the rows correspond to different values of the angle $\alpha^{\prime}$. Thus, one has to conclude that, as expected, a coincidence of the radiating solutions at the far field does not imply that the near fields are also coincidental, see [30].

\section{Stability index method}

Various algorithms for direct and inverse scattering problems require global minimization of functions of many variables, see [30]. Since most objective functions contain many local minima, this is a highly nontrivial task. In several papers, starting with [14], the authors developed and tested the Stability Index Method (SIM) for global minimization. In our presentation here we follow [11], which also contains a convergence analysis and additional numerical results.

The Stability Index Method combines stochastic and deterministic algorithms to find global minima of multidimensional functions. The functions may be nonsmooth and may have multiple local minima. The method examines the change of the diameters of the minimizing sets for its stopping criterion. At first, the algorithm uses the uniform random distribution in the admissible set. Then normal random distributions of decreasing variation are used to focus on probable global minimizers. To test the method, we have applied it to standard test functions of several variables. The computational results show that the SIM is efficient, reliable and robust. 
Table 6

Near field values of two radiating solutions with practically the same far fields

\begin{tabular}{|c|c|c|c|c|}
\hline$\alpha^{\prime}$ & $\operatorname{Re} v_{c}$ & $\operatorname{Im} v_{c}$ & $\operatorname{Re} v$ & $\operatorname{Im} v$ \\
\hline 0.00000 & -1189.60834 & -227.35213 & -0.54030 & -0.84147 \\
\hline 0.31416 & -73.43878 & -15.81270 & -0.58082 & -0.81403 \\
\hline 0.62832 & 1.94958 & 0.19051 & -0.69021 & -0.72361 \\
\hline 0.94248 & 0.03298 & -0.52343 & -0.83217 & -0.55452 \\
\hline 1.25664 & -1.07968 & -0.36021 & -0.95263 & -0.30412 \\
\hline 1.57080 & -1.13445 & 0.00027 & -1.00000 & 0.00000 \\
\hline 1.88496 & -0.96294 & 0.31629 & -0.95263 & 0.30412 \\
\hline 2.19911 & -0.79021 & 0.55436 & -0.83217 & 0.55452 \\
\hline 2.51327 & -0.66472 & 0.71819 & -0.69021 & 0.72361 \\
\hline 2.82743 & -0.59154 & 0.81406 & -0.58082 & 0.81403 \\
\hline 3.14159 & -0.56768 & 0.84565 & -0.54030 & 0.84147 \\
\hline 3.45575 & -0.59154 & 0.81406 & -0.58082 & 0.81403 \\
\hline 3.76991 & -0.66472 & 0.71819 & -0.69021 & 0.72361 \\
\hline 4.08407 & -0.79021 & 0.55436 & -0.83217 & 0.55452 \\
\hline 4.39823 & -0.96294 & 0.31629 & -0.95263 & 0.30412 \\
\hline 4.71239 & -1.13445 & 0.00027 & -1.00000 & 0.00000 \\
\hline 5.02655 & -1.07968 & -0.36021 & -0.95263 & -0.30412 \\
\hline 5.34071 & 0.03298 & -0.52343 & -0.83217 & -0.55452 \\
\hline 5.65487 & 1.94958 & 0.19051 & -0.69021 & -0.72361 \\
\hline 5.96903 & -73.43878 & -15.81270 & -0.58082 & -0.81403 \\
\hline
\end{tabular}

Given a function $f: A \rightarrow \mathbb{R}$, our goal is to minimize it over an admissible set $A$ assumed to be a bounded set in a metric space $X$. Typically, the structure of the objective function $f$ is quite complicated. In particular, it can have many local minima and a nonunique global minimum. To better understand the structure of the minima, let us introduce the minimizing sets $S_{\epsilon}$ of $f$. Let $m=\inf \{f(x): x \in A\}$. Given an $\epsilon>0$ define

$$
S_{\epsilon}=\{x \in A: f(x)<m+\epsilon\},
$$

or

$$
S_{\epsilon}=\left\{x \in A: f(x)<f\left(x_{p}\right)+\epsilon\right\},
$$

if the problem admits a global minimizer $x_{p} \in A$.

Definition. Given an $\epsilon>0$, let $D_{\epsilon}$ be the diameter of the minimizing set $S_{\epsilon}$. We call $D_{\epsilon}$ the Stability Index of the minimization problem (10.1).

We are interested in the behavior of $D_{\epsilon}$ as $\epsilon \rightarrow 0$. So, one can say that the problem (10.1) possesses a set of Stability Indices $\left\{D_{\epsilon}: \epsilon>0\right\}$, and the above definition should be understood in this sense.

One would expect to obtain a stable identification for minimization problems with small (relative to the admissible set) stability indices. Minimization problems with large stability indices either have distinct global minimizers, or the function $f$ is nearly flat in a neighborhood of the global minimizer $x_{p}$. In this situation, and with no additional information known, one has an uncertainty of the minimizer's choice. The stability index provides a quantitative measure of this uncertainty or instability of the minimization.

In a practical minimization problem one constructs a sequence of minimizers $\left\{x_{1}, x_{2}, \ldots\right\} \subset A$, and makes a decision when to terminate the iterations according to a stopping criterion. We assert that the knowledge of the Stability Index provides a valuable tool for the formulation of such a stopping criterion.

Originally, we have applied the Stability Index minimization method to inverse scattering problems arising in quantum mechanical scattering, [14]. Such potential scattering problems are important in quantum mechanics, where they appear in the context of scattering of particles bombarding an atom nucleus. One is interested in reconstructing the scattering potential from the results of a scattering experiment. Assuming a particular structure of the potential, the scattering results can be computed and compared to the given scattering data. Thus the inverse scattering problem is reduced to the minimization of the discrepancy (best fit to data), see [14,31] for details. 
The goal of the SIM algorithm is to find a minimizing set $S_{\epsilon}$ that fits within a small portion of the computational domain $A \subset \mathbb{R}^{N}$. Practically, we assume that $A=[-M, M]^{N} \subset \mathbb{R}^{N}$, for an $M>0$. If it is desirable to introduce different scales for the variables, then the algorithm should be modified accordingly.

Let $0<\delta<1$. The minimization is stable if, given a global minimizer $x_{p}$, we are able to find a minimizing set $S_{\epsilon} \subset C\left[x_{p}, \delta\right]$, where $C\left[x_{p}, \delta\right]$ is the cube centered at $x_{p} \in A$ with the side equal to $2 \delta M$.

The next step is to define a sequence of normal distributions $T_{n}$ with the variances $\mu_{n} \rightarrow 0$, as $n \rightarrow \infty$. Thus we fix an $0<\alpha<1$, and let $\mu_{n}=\alpha^{n}, n=1,2, \ldots$.

Initially, for $n=0$, let the $T_{0}$ be the uniform random distribution in A. A special algorithm SMS, described below, determines a finite Stable Minimizing Set $S_{0} \subset A$. Let $x_{0} \in S_{0}$ be the minimizer in $S_{0}$, that is

$$
f\left(x_{0}\right)=\min \left\{f(x): x \in S_{0}\right\} .
$$

If $S_{0} \subset C\left[x_{0}, \delta\right]$, then the minimization is stable and the global minimizer $x_{p}=x_{0}$.

If, on the other hand, the above inclusion is not achieved, then one continues with another application of the SMS, this time using the normal distribution $T_{1}$ with the mean at $x_{0}$, and the variance $\mu_{1}$, etc. The iterations continue until either $S_{n} \subset C\left[x_{n}, \delta\right]$ or $3 \mu_{n}<2 \delta M$. The last condition is needed to prevent all the trial points to be chosen too close to $x_{n}$, thus preventing a reasonable estimate for the diameter of $S_{n}$.

\section{Stability Index Method (SIM)}

Fix $0<\alpha, \delta<1$. Suppose that $A=[-M, M]^{N}$.

1. Initialization. Let $n=0$. Use the SMS algorithm with the uniform random distribution $T_{0}$ in $A$ to determine the minimizing set $S_{0} \subset A$ and the minimizer $x_{0} \in S_{0}$. Go to the Stopping Criterion (Step 3) to check if additional iterations are needed.

2. ( $n$-th iteration). Let $\mu_{n}=\alpha^{n}$. Use the SMS algorithm with the normal random distribution $T_{n}$ with the mean at $x_{n-1}$ and the variance $\mu_{n}$ to determine the minimizing set $S_{n} \subset A$ and the minimizer $x_{n} \in S_{n}$.

3. Stopping criterion. Let $C\left[x_{n}, \delta\right]$ be the cube centered at $x_{n} \in A$ with the side equal to $2 \delta M$.

If $S_{n} \subset C\left[x_{n}, \delta\right]$, then stop. The minimization is stable. The estimated global minimizer $x_{p}$ is $x_{n}$.

If $S_{n} \not \subset C\left[x_{n}, \delta\right]$ and $3 \mu_{n}<2 \delta M$, then stop. The minimization is unstable. The diameter (Stability Index) $D_{n}$ of $S_{n}$ is a measure of the instability of the minimization.

Otherwise, increase $n$ by 1 , and return to Step 2 to do another iteration.

Note that the obtained point $x_{p}$ is an estimated global minimizer.

The main part of the Stability Index Method is the SMS algorithm which determines stable minimizing sets $S_{n}$, corresponding to the random distributions $T_{n}$. These distributions are either uniform in $A$ or normal with a given variation $\mu_{n}$.

The SMS algorithm is an iterative algorithm. It can be called an Iterative Reduced Random Search method. Choose an integer $K>0$ assuming that $K$ random points in $S_{\epsilon}$ are sufficient to estimate its diameter $D_{\epsilon}$. If $n \geq 1$, then the calling algorithm SIM provides the minimizing set $S_{n-1}$, its minimizer $x_{n-1}$, and the variance $\mu_{n}$.

Let a batch $H^{1} \subset A$ of $L>K$ trial points be generated in the admissible set $A$ according to the random distribution $T_{n}$. If $n=0$, then $T_{0}$ is just the uniform random distribution in $A$. If $n \geq 1$, then $T_{n}$ is the normal distribution with the variance $\mu_{n}$, and the mean at $x_{n-1}$. Let $Q_{U}^{1}$ be the subset of $K$ points from $H^{1}$ where the objective function $f$ attains its $K$ smallest values. That is

$$
\max \left\{f\left(u_{i}\right): u_{i} \in Q_{U}^{1}\right\} \leq \min \left\{f\left(u_{i}\right): u_{i} \notin Q_{U}^{1}\right\} .
$$

Use each point $u_{i} \in Q_{U}^{1}$ as the initial guess for a Local Minimization Method (LMM) of your choice, e.g. the conjugate gradient method, etc. The specific LMM used by us is described below. While the use of a local minimization is not, strictly speaking, necessary for the SIM, it provides a significant improvement in the performance of the algorithm, and is highly recommended. Thus for each starting point $u_{i} \in Q_{U}^{1}$ the LMM produces a minimizer $v_{i} \in A$. Let $Q_{V}^{1}$ be the set of all such minimizers. Let $Q^{1}$ be the subset of $Q_{U}^{1} \cup Q_{V}^{1}$ containing $K$ points with the smallest values of $f$, and $q^{1}$ be the minimizer in $Q^{1}$. Define the radius of $Q^{1}$ by

$$
R^{(1)}=\max \left\{\left\|z_{i}-q^{1}\right\|: z_{i} \in Q^{1}, i=1,2, \ldots, K\right\} .
$$


The idea of the Stability Index Method is to iteratively construct subsets $Q^{j}$ until their diameters are stabilized. Practically, one can achieve the same goal by estimating and examining the radius $R^{(j)}$ of the set $Q^{j}$. This also requires less computational effort.

To construct the next set $Q^{2}$ generate another batch $H^{2} \subset A$ of $L$ trial points according to the uniform random distribution, if $n=0$, or, for $n \geq 1$, according to the normal distribution $T_{n}$ with the variance $\mu_{n}$, and the mean at $q^{1}$. Let $Q_{U}^{2}$ be the subset of $K$ points from $H^{2} \cup Q^{1}$ having the smallest $K$ values of $f$. Apply the LMM to produce the set of minimizers $Q_{V}^{2}$. Of course, if some point $u_{i} \in Q_{U}^{2}$ has already been used as an initial guess for the LMM in the previous iteration, it is excluded from the LMM application. Let $Q^{2}$ be the subset of $Q_{U}^{2} \cup Q_{V}^{2}$ containing $K$ points with the smallest values of $f$. Let $q^{2}$ be the minimizer in $Q^{2}$, and $R^{(2)}=\max \left\{\left\|z_{i}-q^{2}\right\|: z_{i} \in Q^{2}, i=1,2, \ldots, K\right\}$ be its radius, etc.

This way one produces a sequence of the minimizing sets $Q^{j}, j=1,2, \ldots$ Let $0<\gamma<1$, and $P$ be a positive integer. The iterations are terminated if the maximum number of iterations $N_{\max }$ is exceeded or the following Stopping Criterion is satisfied:

$$
\left|R^{(j)}-\frac{1}{P} \sum_{i=j-P+1}^{j} R^{(i)}\right|<2 \gamma M .
$$

In either case, when the last iteration $j$ is determined from (10.6) or $j=N_{\max }$, we let $S_{n}=Q^{j}$ and $x_{n}=q^{j}$.

Stable Minimizing Set (SMS) algorithm

Fix $0<\gamma<1$, and integer $K, L>K, P, N_{\max }$. Constant $M$, normal random distribution $T_{n}$, its variance $\mu_{n}$ (for $n \geq 1$ ), the minimizing set $S_{n-1}$, and the minimizer $x_{n-1}$ are supplied by the calling algorithm SIM.

1. Initialization. Let $j=1$.

- For $n=0$. Generate a batch $H^{1}$ of $L$ trial points in $A \subset \mathbb{R}^{N}$ using the uniform random distribution. Let $Q_{U}^{1}$ be the subset of $K$ points from $H^{1}$ where the objective function $f$ attains its $K$ smallest values. Go to Step 4.

- For $n \geq 1$. Generate a batch $H^{1}$ of $L$ trial points in $A \subset \mathbb{R}^{N}$ using the normal distribution $T_{n}$ with the variance $\mu_{n}$ and the mean at $x_{n-1}$. Let $Q_{U}^{1}$ be the subset of $K$ points from $H^{1} \cup S_{n-1}$ where the objective function $f$ attains its $K$ smallest values. Go to Step 4 .

2. Iterative step $(j \geq 2)$.

- For $n=0$. Generate a batch $H^{j}$ of $L$ trial points in $A \subset \mathbb{R}^{N}$ using the uniform random distribution.

- For $n \geq 1$. Generate a batch $H^{j}$ of $L$ trial points in $A \subset \mathbb{R}^{N}$ using the normal distribution $T_{n}$ with the variance $\mu_{n}$ and the mean at $q^{j-1}$.

3. Let $Q_{U}^{j}$ be the subset of $K$ points from $H^{j} \cup Q^{j-1}$ where the objective function $f$ attains its $K$ smallest values.

4. Local minimization. Use each unflagged point $u_{i} \in Q_{U}^{j}$ as the initial guess for a Local Minimization Method

(LMM). Let $v_{i} \in A$ be the resulting minimizer. Let $Q_{V}^{j}$ be the set of all such minimizers resulting from the application of LMM to $Q_{U}^{j}$. Flag all points in $Q_{U}^{j}$ and $Q_{V}^{j}$.

5. Let $Q^{j}$ be the subset of $Q_{U}^{j} \cup Q_{V}^{j}$ containing $K$ points with the smallest values of $f$ and $q^{j}$ be the minimizer in $Q^{j}$. Define the radius of $Q^{j}$ by

$$
R^{(j)}=\max \left\{\left\|z_{i}-q^{j}\right\|: z_{i} \in Q^{1}, i=1,2, \ldots, K\right\} .
$$

6. Stopping criterion.

- If $j<P$, increase $j$ by 1 and return to Step 2 for another iteration.

- If $j \geq P$, compute the average radius during the last $P$ iterations:

$$
R_{a}=\frac{1}{P} \sum_{i=j-P+1}^{j} R^{(i)} .
$$

- Termination. If $\left|R^{(j)}-R_{a}\right| \leq 2 \gamma M$, or $j \geq N_{\max }$, let $S_{n}=Q^{j}, x_{n}=q^{j}$ and exit the procedure.

- Otherwise, increase $j$ by 1 and return to Step 2 for another iteration.

The SMS implementation involves a combination of stochastic (global) and deterministic (local) minimization methods. Generally, local searches offer more precision and speed than their global counterparts, so that adding a local step to a global minimization algorithm should yield improvement in both areas. Likewise, by itself, a local 
minimization method will very often produce points of considerable distance from the actual global minimizer, that is it would be trapped in one of many local minima of the objective function $f$. Adding a global step helps the algorithm escape from local minima, and explore the entire admissible set $A$. The use of various normal distributions of decreasing variance is similar to ideas of the simulated annealing method [19].

The particular Local Minimization Method (LMM) used in the numerical experiments was a modification of Powell's minimization method in $\mathbb{R}^{N}$, [7]. It was chosen with applications in mind, for which the objective function $f$ does not have a convenient expression for its gradient. Either a Golden Search or Brent's method can be used for one dimensional minimizations, [24].

\section{Modified Powell's method}

1. Choose the set of directions $u_{i}, i=1,2, \ldots, N$, to be the standard basis in $\mathbb{R}^{N}$

$$
u_{i}=(0,0, \ldots, 1, \ldots, 0),
$$

where 1 is in the $i$-th place.

2. Save the starting point $p_{0}$.

3. For $i=1, \ldots, N$ move from $p_{i-1}$ along the direction $u_{i}$ and find the point of minimum $p_{i}$.

4. Set $v=p_{N}-p_{0}$.

5. Move from $p_{0}$ along the direction $v$ and find the minimum. Call it $p_{0}$ again. It replaces $p_{0}$ from Step 2.

6 . Repeat the above steps until a stopping criterion is satisfied. The resulting point is $p_{\min }$.

Note that $f\left(p_{\min }\right) \leq f\left(p_{0}\right)$ for any objective function $f$ used in the Local Minimization Method.

\section{Numerical results for SIM}

The Stability Index Method described in the previous sections was tested on several functions designed to test and compare various minimization algorithms, see [11] for additional test functions results. The experiments were conducted on a $2.8 \mathrm{GHz}$ PC with $256 \mathrm{MB}$ RAM.

In all the numerical experiments we used the same parameter values: $\alpha=0.8, \delta=0.001, \gamma=0.001, K=$ $30, L=5000, P=6$, and $N_{\max }=30$. For each test function the admissible set $A$ is a cube $[-M, M]^{N}$ provided in the function's description together with its global minimizer.

Test function 1

$$
f(x, y)=\left(\sum_{i=1}^{5} i \cos [(i+1) x+i]\right)\left(\sum_{i=1}^{5} i \cos [(i+1) y+i]\right)+0.5\left((x+1.4213)^{2}+(y+0.80032)^{2}\right) .
$$

The minimum is sought on $[-5,5] \times[-5,5]$. This function has a global minimum at $(-1.42513,-0.80032)$ with a function value of -186.73091 , [37].

Test function 2

$$
f(x, y)=\mathrm{e}^{\sin (50 x)}+\sin \left(60 \mathrm{e}^{y}\right)+\sin (70 \sin x)+\sin (\sin (80 y))-\sin (10(x+y))+\left(x^{2}+y^{2}\right) / 4 .
$$

The minimum is sought on $[-1,1]^{2}$. According to [6] the minimum occurs at approximately $(-0.0244031,0.2106124)$ with a function value of -3.30686865 .

Test function 3

$$
f(x)=\frac{\pi}{N}\left(10 \sin ^{2}\left(\pi y_{1}\right)+\sum_{i=1}^{N-1}\left(y_{i}-1\right)^{2}\left(1+10 \sin ^{2}\left(\pi y_{i}+1\right)\right)+\left(y_{N}-1\right)^{2}\right)
$$

where $x=\left(x_{1}, x_{2}, \ldots, x_{N}\right) \in \mathbb{R}^{N}, y_{i}=1+0.25\left(x_{i}-1\right), i=1,2, \ldots, N$. The minimum is sought on $[-10,10]^{N}$. This function has a global minimum at $x=(1,1, \ldots, 1)$ with a function value of 0 , [37].

The results of the minimization using the SIM for these test functions are shown in Table 7. The algorithm was run 20 times for each function. It found the correct global minimum most of the time. The "success rate" column in Table 7 shows the percentage of trials in which the global minimum was found exactly. The "Function evaluation" column shows the average number of times the objective function was evaluated. Finally, Table 7 shows the average run time, in seconds, for a single trial run. 
Table 7

Results of the computational experiments for SIM

\begin{tabular}{lccccc}
\hline Function & Dimension $N$ & Actual minimum & Found minimum & Success rate (\%) & Average run time (s) \\
\hline 1 & 2 & -186.731 & -186.731 & 100 & 2 \\
2 & 2 & -3.30687 & -3.30687 & 100 & 2 \\
3 & 5 & 0.00000 & 0.00000 & 100 & 7 \\
3 & 10 & 0.00000 & 0.00000 & 100 & 16 \\
3 & 20 & 0.00000 & 0.00000 & 100 & 50 \\
\hline
\end{tabular}

\section{Conclusions}

Let $D$ be a 2D or 3D obstacle, $S$ be its boundary, and $u_{0}$ be the incident field. Rayleigh conjectured that the acoustic field $u$ in the exterior of the obstacle is given by

$$
u(x, \alpha)=\mathrm{e}^{\mathrm{i} k \alpha \cdot x}+\sum_{\ell=0}^{\infty} A_{\ell}(\alpha) \psi_{\ell}, \quad \psi_{\ell}:=Y_{\ell}\left(\alpha^{\prime}\right) h_{\ell}(k r), \alpha^{\prime}=\frac{x}{r} .
$$

While this conjecture (RC) is false for many obstacles, it has been modified to obtain a representation for the solution of (1.1) and (1.2) and to obtain its error.

It is proved that if $v_{\epsilon}$ is an outgoing solution of the Helmholtz equation in the exterior domain $D^{\prime}$ and $u_{0}+v_{\epsilon}$ approximates zero in $L^{2}(S)$-norm on the boundary $S$, then $v_{\epsilon}$ approximates the exact scattered field $v$ in $D^{\prime}$, see Theorems 2.1 and 2.3. The modified Rayleigh conjecture approach to obstacle scattering problems is based on the following observation: the functions $\psi_{\ell}(x, z), z \in D$ and their linear combinations are outgoing solutions to the Helmholtz equation in the exterior domain. Therefore, one just needs to find a combination of such functions that gives the best fit to $-u_{0}$ on the boundary $S$. Then this combination approximates the scattered field everywhere in the exterior $D^{\prime}$ of the obstacle $D$ and the error of this approximation is given in Theorems 2.1 and 2.3.

In this paper we describe several implementations of the MRC method which give an efficient approach to solving obstacle scattering problems for 2D and 3D problems with complicated geometries. Our implementations of the MRC method worked more efficiently than the BIEM method.

Various methods for solution of direct and inverse scattering problems require a global minimization of the objective function. We developed the Stability Index Method which is a robust and efficient algorithm for global minimization. Its efficiency comes from a combined use of global and local minimization. The global (stochastic) part employs uniform and normal random distributions. It can be combined with local (deterministic) methods appropriate for the objective function. The diameters of the minimizing sets (Stability Index) are used for a self-contained stopping criterion. The computational experiments show that the method was successful for various standard test functions over multidimensional domains. No adjustment of parameters was needed in different tests. The method is well suited for low dimensional minimization problems. Its performance deteriorates for higher dimensional problems. The Stability Index Method is a valuable addition to already existing global minimization methods.

\section{References}

[1] H.D. Alber, A quasi-periodic boundary value problem for the Laplacian and the continuation of its resolvent, Proc. Roy. Soc. Edinburgh Sec. A $82(3-4)(1978-79)$ 251-272.

[2] N.C. Albertsen, J.-M. Chesneaux, S. Christiansen, A. Wirgin, Comparison of four software packages applied to a scattering problem, Math. Comput. Simulation 48 (1999) 307-317.

[3] R. Barantsev, Concerning the Rayleigh hypothesis in the problem of scattering from finite bodies of arbitrary shapes, Vestn. Lenungrad Univ. Math. Mech. Astron. 7 (1971) 56-62.

[4] A. Bonnet-Bendhia, Guided waves by electromagnetic gratings and non-uniqueness examples for the diffraction problem, Math. Appl. Sci. 17 (1994) 305-338.

[5] A. Bonnet-Bendhia, K. Ramdani, Diffraction by an acoustic grating perturbed by a bounded obstacle, Adv. Comput. Math. 16 (2002) 113-138.

[6] F. Bornemann, D. Laurie, S. Wagon, J. Waldvogel, The SIAM 100-Digit Challenge: A Study in High-Accuracy Numerical Computing, Society for Industrial and Applied Mathematics, Philadelphia, 2004.

[7] P. Brent, Algorithms for Minimization without Derivatives, Prentice-Hall, Englewood Cliffs, NJ, 1973.

[8] S. Christiansen, R.E. Kleinman, On a misconception involving point collocation and the Rayleigh hypothesis, IEEE Trans. Antennas Propagation 44 (10) (1996) 1309-1316. 
[9] D. Colton, R. Kress, Inverse Acoustic and Electromagnetic Scattering Theory, Springer-Verlag, New York, 1992.

[10] A. Doicu, Y. Eremin, T. Wriedt, Acoustic and Electromagnetic Scattering Analysis Using Discrete Sources, Academic Press, London, 2000.

[11] J. Dover, S. Gutman, Stability index method for global minimization, J. Global Optim. 36 (2006) 307-318.

[12] D.M. Eidus, Some boundary-value problems in infinite regions, Izvestia Akademii Nauk SSSR, Ser. Math. 27 (1963) $1055-1080$.

[13] S. Gutman, A.G. Ramm, Numerical implementation of the MRC method for obstacle scattering problems, J. Phys. A: Math. Gen. 35 (2002) 8065-8074.

[14] S. Gutman, A.G. Ramm, W. Scheid, Inverse scattering by the stability index method, J. Inverse Ill-Posed Problems 10 (N5) (2002) 487-502.

[15] S. Gutman, A.G. Ramm, Support function method for inverse scattering problems, in: A. Wirgin (Ed.), Acoustics, Mechanics and Related Topics of Mathematical Analysis, World Scientific, New Jersey, 2003, pp. 178-184.

[16] S. Gutman, A.G. Ramm, Modified Rayleigh Conjecture Method with optimally placed sources, J. Appl. Funct. Anal. 1 (N2) (2006) $223-236$.

[17] L. Kazandjian, Rayleigh-Fourier and extinction theorem methods applied to scattering and transmission at a rough solid-solid interface, J. Acoust. Soc. Amer. 92 (1992) 1679-1691.

[18] L. Kazandjian, Comments on "Reflection from a corrugated surface revisited", [J. Acoust. Soc. Amer. 96 (1994) 1116-1129], J. Acoust. Soc. Amer. 98 (1995) 1813-1814

[19] S. Kirkpatrick, Optimization by simulated annealing: Quantitative studies, J. Statist. Phys. 34 (1984) 975-986.

[20] J.L. Lions, E. Magenes, Non-Homogeneous Boundary Value Problems and Applications, Springer, New York, 1972.

[21] R. Millar, On the Rayleigh assumption in scattering by a periodic surface, Proc. Cambridge Philos.Soc. 69 (1971) 217-225. 65 (1969) 773-791.

[22] R. Millar, The Rayleigh hypothesis and a related least-squares solution to the scattering problems for periodic surfaces and other scatterers, Radio Sci. 8 (1973) 785-796.

[23] R. Petit (Ed.), Electromagnetic Theory of Gratings, in: Topics in Current Physics, vol. 22, Springer-Verlag, Berlin, New York, 1980.

[24] W.H. Press, S.A. Teukolsky, W.T. Vetterling, B.P. Flannery, Numerical Recipes in FORTRAN, second ed., Cambridge University Press, 1992.

[25] A.G. Ramm, Multidimensional Inverse Scattering Problems, Longman/Wiley, New York, 1992.

[26] A.G. Ramm, Multidimensional Inverse Scattering Problems, Mir, Moscow, 1994 Expanded Russian edition of [25].

[27] A.G. Ramm, Modified Rayleigh conjecture and applications, J. Phys. A: Math. Gen. 35 (2002) L357-L361.

[28] A.G. Ramm, Inverse Problems, Springer, New York, 2005.

[29] A.G. Ramm, S. Gutman, Modified Rayleigh conjecture for scattering by periodic structures, Int. J. Appl. Math. Sci. 1 (N1) (2004) 55-66.

[30] A.G. Ramm, S. Gutman, Numerical solution of obstacle scattering problems, Intern. J. Appl. Math. Mech. 1 (2005) 1-32.

[31] A.G. Ramm, S. Gutman, Optimization methods in direct and inverse scattering, in: V. Jeyakumar, A.M. Rubinov (Eds.), Continuous Optimization: Current Trends and Modern Applications, Springer, New York, 2005, pp. 51-110.

[32] A.G. Ramm, W. Chen, Numerical method for solving obstacle scattering problems by an algorithm based on the modified Rayleigh conjecture, Int. J. Appl. Math. Sci. 2 (N1) (2005) 11-21.

[33] A.G. Ramm, Scattering by Obstacles, D. Reidel Publishing Co., Dordrecht, 1986.

[34] A.G. Ramm, Wave Scattering by Small Bodies of Arbitrary Shapes, World Sci. Publ., Singapore, 2005.

[35] A.G. Ramm, Modified Rayleigh conjecture for static problems, Appl. Math. Lett. 18 (N12) (2005) 1396-1399.

[36] J.W. Rayleigh, On the dynamical theory of gratings, Proc. Roy. Soc. A 79 (1907) 399-416.

[37] K.F.C. Yiu, Y. Liu, K.L. Teo, A hybrid descent method for global optimization, J. Global Optim. 28 (2004) $229-238$. 